\title{
Alanine Metabolism in Bacillus subtilis
}

\author{
Karzan R. Sidiq ${ }^{1,2 *}$, Man W. Chow ${ }^{1 *}$, Zhao Zhao ${ }^{1}$ and Richard A. Daniel ${ }^{1 * *}$ \\ ${ }^{1}$ Centre for Bacterial Cell Biology, Institute for Cell and Molecular Biosciences, Medical \\ Faculty, Newcastle University, Newcastle Upon Tyne, NE2 4AX, UK \\ ${ }^{2}$ Current address: College of Health and Applied Sciences, Charmo University, 46023 \\ Chamachamal, Sulaimani, Kurdistan Region-IRAQ.
}

* Equal contributions were made by these authors

** Corresponding author: Richard A. Daniel, Centre for Bacterial Cell Biology, Institute for Cell and Molecular Biosciences, Medical Faculty, Newcastle University, Newcastle Upon Tyne, NE2 4AX, UK. richard.daniel@newcastle.ac.uk, Tel: +44 (0) 191208 3239, Fax: +44 (0) 191222 $\underline{7424 .}$ 
bioRxiv preprint doi: https://doi.org/10.1101/562850; this version posted April 18, 2019. The copyright holder for this preprint (which was not certified by peer review) is the author/funder. All rights reserved. No reuse allowed without permission. 


\begin{abstract}
:
Alanine plays a crucial role in bacterial growth and viability, the L-isomer of this amino acid is one of the building blocks for protein synthesis, and the D-isomer is incorporated into the bacterial cell wall. Despite a long history of genetic manipulation of Bacillus subtilis using auxotrophic markers, the genes involved in alanine metabolism have not been characterised fully. Here we identify a B. subtilis alanine permease, $Y \operatorname{tn} A(D a t A)$, which has a major role in the assimilation of $D$-alanine from the environment, and provide an explanation for the observation that growth of $B$. subtilis does not result in the significant accumulation of extracellular D-alanine. Interestingly, this transporter seems to have specificity for D-alanine but also transports L-alanine. We also show that, unlike $E$. coli where multiple enzymes have a biochemical activity that can generate alanine, in B. subtilis the primary synthetic enzyme for alanine is encoded by alaT, although a second gene, dat, is present that can support slow growth of an L-alanine auxotroph. However, Dat probably synthesises D-alanine and its activity is influenced by the abundance of L-alanine. Our work provide a clear insight into the complex network of alanine metabolism, and also points to the possibility that the relative abundance of D- and L-alanine might be linked with cytosolic pool of D and L-glutamate, coupling protein and cell envelope synthesis with the metabolic status of the cell.
\end{abstract}




\section{Author Summary}

D-alanine is a central component of the cell envelope of Bacillus subtilis, it has an essential role in both peptidoglycan crosslinking and is also incorporated into the teichoic acids. Growth of the bacterial cell results in the release $D$-alanine and as this process is on the outside of the cell it should result in the accumulation of this amino acid in the culture medium. However, this has not been see to occur if $B$. subtilis cultures. This work identifies and characterises a membrane transporter as having primary role seems to be the assimilation of D-alanine as it is released from the cell wall. The analysis shows that in vivo this transporter is specific for alanine, but appears to have greater affinity for the D-isomer of this amino acid. This work also clarifies the genetics of alanine biosynthesis in B. subtilis through which there are interesting difference with other bacterial species.

\section{Introduction}

Bacterial cell growth requires the coordinated synthesis of all the metabolites necessary for the enlargement of the cell. One essential component of the bacterial cell wall that is unique to bacteria is D-alanine. This amino acid isomer is universally used by bacteria in the synthesis of the peptidoglycan (PG) as a substrate for penicillin binding protein-mediated crosslinking of the glycan strains $(1,2)$. In Gram-positive bacteria, it is also used to modify the teichoic acids, which are present as a second major component of the cell wall and membrane $(3,4)$.

Bacteria obtain D-alanine primarily by converting L-alanine by racemization. L-alanine, on the other hand, can be biosynthesised by the cell, in addition to assimilation from outside the cell and racemization of $\mathrm{D}$-alanine. Alanine synthesis and metabolism is increasingly being exploited for antibacterial and industrial applications. For example, exogenous L-alanine has been shown to increase the susceptibility of some pathogens to antibiotics (5); D-alanine can inhibit germination of the spores of Bacillus and Clostridium species by acting as an competitor of L-alanine, one of the most effective 
germinants $(6,7)$; the level of D-alanine has also been shown to be able to alter the course of infection by Bacillus anthracis (8). In B. subtilis an alanine racemase gene has been utilised for plasmid maintenance (9), and further explored by Xia et al. (2007) to replace antibiotics resistance genes in the construction of a food-grade expression system (10).

It has long been proposed that growth of Gram-positive rod shaped bacteria results from the degradation of the outer old cell wall material (11-13) and the release of this material into the growth media. It is also known that formation of each cross-link in the peptidoglycan will result in the release of a D-alanine residue. PG peptide side chains that do not take part in cross-links are also matured soon after synthesis in B. subtilis, with both of the terminal D-alanine residues being cleaved off sequentially $(14,15)$. D-alanine is also released from the teichoic acids by spontaneous hydrolysis (16), consequently there should be an accumulation of free D-alanine residues in the vicinity of a growing cell unless actively re-assimilated by the bacterial cells. Pervious analyses of culture media composition after the growth of various bacterial species have revealed that many release D-amino acids on reaching stationary phase growth, presumably as secondary metabolites or signalling molecules (2). Indeed, stationary phase cultures of $S$. aureus have been shown to have significant levels of D-alanine present (1). Surprisingly, however, analysis of spent media from B. subtilis cultures were essentially devoid of Dalanine (1). Since both bacterial species incorporate this amino acid into the cell envelope in similar ways this suggested that $B$. subtilis has an efficient recycling system for D-alanine.

In B. subtilis the biosynthetic pathways for alanine are poorly characterised, and this is complicated by the partial annotation and functional characterisation of the racemase and aminotransferase genes (17). In B. subtilis two alanine racemases, AlrA (originally called Dal) (18) and YncD (also called Alr) (19), have been identified and it seems that YncD/Alr only functions in sporulation. B. subtilis also encodes two putative aminotransferases: YugH which is also called AlaT (20), and YheM (Dat) which is a homolog of the Bacillus sphaericus Dat protein, a D-amino acid transaminase that 
converts D-alanine to D-glutamate (21). Alanine degradation, on the other hand, is believed to be carried out by Ald, an alanine dehydrogenase that reversibly catalyses the reductive amination of alanine to pyruvate. Ald is required for utilisation of alanine as a sole carbon or nitrogen source $(22,23)$ and also for sporulation in B. subtilis (24). A recent characterisation of the roles of DacA/LdcB (15) in $B$. subtilis has indicated that there is a high demand for the D-isomer of alanine for cell envelope biosynthesis, as might be expected considering its presence in both peptidoglycan and teichoic acids. Dalanine can be supplied by either cytosolic isomerisation of alanine by the action of AlrA (18) or assimilation from the environment, although this isomeric form is not normally available in nature in significant concentrations. Like glutamate, alanine is abundant in the cytoplasm of growing cells, present in both the L- and D- isomeric form and there is strong evidence for the presence of mechanisms for precise control of the concentration of these amino acids and presumably their isomeric state (25). Surprisingly, in contrast to glutamate, the biosynthetic pathway for alanine is poorly defined in $B$. subtilis. Also, little is known about the uptake mechanisms for the assimilation of exogenous alanine by bacteria. Although many transporters are bioinformatically annotated as being potentially involved in alanine uptake, experimental evidence is limited and only one from E. coli has been implicated in Dalanine transport (26).

Here we report the identification of an alanine transporter, which we have named DatA (ㅁ-

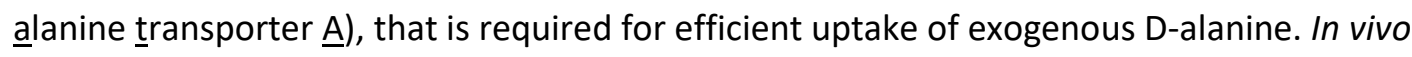
characterisation shows that DatA has specificity for alanine, with preference for D-alanine, and that it does not seem to function in the uptake of other amino acids. We have also clarified aspects of the synthetic pathways for both isomeric forms of alanine in B. subtilis. Our results provide explanations for some longstanding observations; we show that AlaT (YugH) functions as the major transaminase responsible for the biosynthesis of alanine, whereas the second enzyme Dat has a minor role in alanine 
biosynthesis, probably functioning in synthesis of D-alanine and its activity is inhibited by the presence of L-alanine.

\section{Results and Discussion:}

\section{Growth characteristics and L-alanine sensitivity of a D-alanine dependent strain of B. subtilis.}

Deletion of the alanine racemase gene, alrA (dal), has previously been shown to result in auxotrophy for

D-alanine (9). We sought to use this property to determine if a specific D-alanine uptake system was

present or if uptake was mediated by transporters with degenerate specificity as has been suggested for some other amino acid uptake systems (27). So we characterised the growth of a strain lacking the conditionally essential alanine racemase, alrA (strain RD180) in a LB growth medium. As shown by Ferrari et al. (9), in the absence of added D-ala the strain failed to grow in the medium, and no to poor growth was observed in the presence of less than $200 \mu \mathrm{M}$ D-alanine. But when supplemented with Dalanine up to $0.5 \mathrm{mM}$ the strain exhibited concentration dependent growth. Above $0.5 \mathrm{mM}$ D-alanine the strain grew maximally irrespective of the D-alanine concentration (Fig $1 A$ and $B$ ), showing that the strain was able to take up D-alanine efficiently enough to support growth. However, even at the highest concentration the resulting growth rate was significantly lower than that of the wild type strain (168CA) (Fig 1B), suggesting that uptake was not sufficient to supply the demands of normal growth. From these growth curves it was determined that $450 \mu \mathrm{M}$ D-alanine was sufficient for the strain to grow optimally. We then used a fixed D-alanine concentration $(450 \mu \mathrm{M})$ and supplemented the culture medium (LB) with different concentrations of other amino acids to determine if the presence of an excess of one particular amino acids would interfere specifically with the growth of the alrA null strain, and not the wild type strain 168CA. On testing all 20 amino acids it was found that only the addition of excess Lalanine resulted in any significant deviation in the growth of the alrA null strain but not for 168CA (Fig. 1C). None of the other amino acids tested had any significant effect on the growth of the strains and 
gave essentially identical growth curves to those that are shown in Fig. $1 \mathrm{C}$ for glycine and proline, apart from cysteine that at elevated concentrations was toxic to both strains (data not shown). This result indicated that the D-ala uptake perturbed specifically by L-alanine but no other amino acids, suggesting the existence of an alanine specific transporter and that L-alanine competitively interfered with the uptake of the D-isomer.

As the abundance of amino acids in LB is not well defined we used the analyses of individual media components (28) to estimate the free L-alanine concentration in LB medium to be approximately $4 \mathrm{mM}$. This was approximately 10 fold higher than the concentration of D-alanine that we used (450 $\mu \mathrm{M})$, thus it was possible that the reduced growth rate observed of the D-alanine dependent strain (alrA null) was caused by the presence of excess L-alanine. To test this possibility the analysis was repeated using a defined medium (GM) where all amino acids except L-ala were present at $1 \mathrm{mM}$ and $\mathrm{D}$-ala was absent or present at 1 or $0.5 \mathrm{mM}$. The resulting growth of the alrA null strain (RD180) and the wild-type was monitored over time (Fig 2A and B). As seen previously growth of RD180, with $0.5 \mathrm{mM}$ D-alanine permitting growth comparable to that seen for the wild type strain in the same medium (Fig 2, A and B). Here the slower growth of RD180 compared to the wild type was not seen, presumably because the "maximal" growth rate in this medium did not exceed the rate at which D-alanine could be taken up by the auxotroph.

Using the same defined medium (GM) with D-alanine present at $1 \mathrm{mM}$, the culture medium was supplemented with L-alanine at 5, 10, 20 and $40 \mathrm{mM}$ and the growth of RD180 was compared with that seen for the wild type (Fig $2 \mathrm{D}$ and E). Again, when the L-alanine concentration in the medium was greater than 10 times that of D-ala growth of RD180 was perturbed, and where the L-alanine was present at high concentrations ( 40 fold excess) the RD180 failed to grow.

It has previously been proposed that the presence of L-alanine might alter metabolism such that D-alanine is metabolised efficiently into pyruvate and so may not be available for cell wall synthesis, 
resulting in growth inhibition (23). However, it was equally possible that the transporter of exogenous alanine was unable to differentiate between the isomeric states of alanine and consequently, when the ratio of extracellular L- to D-alanine exceeded a certain level, D-ala uptake was insufficient to permit cell envelope synthesis (peptidoglycan and teichoic acid synthesis) due to transport competition. Strangely, however, as has been noted previously $(9,23), \operatorname{RD} 180$ when inoculated on equivalent minimal solid medium was found to grow in the absence of D-alanine, albeit at a slower rate than in the presence of D-alanine (Fig 3). This indicates that under these conditions an alternate, less efficient pathway for the synthesis of D-ala was occurring. Interestingly, we found that this growth was also specifically inhibited if L-alanine is present (Fig. 3B) in the medium, suggesting that L-ala repressed the alternate $\mathrm{D}$-alanine synthetic pathway.

\section{Identification of an alternate D-alanine synthetic pathway.}

To try and identify the genes involved in this second synthetic pathway for D-alanine we firstly eliminated the possibility that the sporulation racemase, $\operatorname{alrB}(19)$, might be expressed under these conditions. We introduced the alrA null mutation into a strain already deleted for alrB (Fig. 3B) and found that the alrA alrB double mutant was still able to grow on GM plates in the absence of D-alanine, comparable to that seen for the alrA single mutant (Fig. 3B). We next introduced the alrA null mutation into strains deleted for known non-essential racemase-like genes (yitF (29), yrpC (30) or racX (31)). Again all of these double mutants were able to grow slowly on GM plates without D-alanine (Fig. 3A). We then tested other genes known or inferred to function in alanine metabolism, including the alanine dehydrogenase ald (24) and the putative aminotransferase genes yugH (alaT) and dat (21), to see if they were required in a strain lacking alrA.

The first of these genes, $y u g H$, is annotated as being similar to a PLP-dependent aspartate aminotransferase. Although it has not yet been characterised fully, it has been suggested to be involved 
in L-alanine biosynthesis, functioning as a transaminase and named alaT (A.L. Sonenshein; unpublished;

(20)). Our analysis revealed that the alaT null mutant was auxotrophic for L-alanine and further characterisation of this gene is described later in this paper. Null mutations for either dat or ald could be easily introduced into the alrA mutant provided that exogenous D-alanine was present. These double mutants were then tested for the ability to grow on minimal media in the absence of D-alanine. Strain KS79, deleted for both alrA and ald, was able to grow, but a strain lacking both alrA and dat (strain KS78) was not able to propagate in the absence of D-alanine even with prolonged incubation (Fig 3B). Further analysis of the growth of this strain showed that its growth was dependent upon exogenous D-alanine under all growth conditions we have tested. Therefore, in the absence of AlrA the putative aminotransferase Dat is implicated in the synthesis of D-alanine, though at a reduced rate. Interestingly, this double mutant seemed more sensitive to the relative concentrations of D- and L-alanine in liquid culture compared to the alrA single mutant (Figure 2, panels C, F and I).

These results have interesting parallels with Listeria monocytogenes where an aminotransferase, with significant similarity to Dat of B. subtilis, has been shown to function in the synthesis of D-alanine when the supposed essential racemase was deleted (32). However, unlike $B$. subtilis, the mutant strains of L. monocytogenes described do not seem to exhibit any sensitivity to Lalanine. This suggested that either the expression or the biochemical activity of Dat is significantly different in this related bacterial species. This possibility is further supported by the recent characterisation of a similar enzyme in Mycobacterium smegmatis (33) where a Dat homologue was found to be able to compensate for null mutations is either the alanine racemase or the glutamate racemase null mutation. This property indicates that at least in $M$. smegmatis the enzymatic activity is bidirectional.

\section{Identification of the D-alanine uptake system}


To determine if a gene encoding a D-ala specific transporter exists, a simple synthetic lethal screen was designed, using the alrA null mutant and systematically introducing null mutations for genes predicted to encode proteins involved in transport of solutes across the membrane. If $D$-alanine uptake was perturbed by the introduction of this second mutation the resulting double mutant would be inviable. In an initial screen a collection of 43 knockouts of transporter-like genes in the form of pMUTIN integrations (34) were introduced individually into the alrA null background, and the transformants selected for on nutrient agar with $1 \mathrm{mg} / \mathrm{ml}$ erythromycin and supplemented with $0.5 \mathrm{mM}$ D-alanine. Most of those tested were found to transform into the strain efficiently; the resulting colonies had both of the expected antibiotic resistance markers and were dependent upon exogenous D-alanine. However, 3 knockout mutations, corresponding to $y f k T, y d g F$ and $y \operatorname{tn} A$, gave rise to very few colonies on selective plates and all of which were found to have lost the alrA null mutation, suggesting that the loss of either of these genes in combination with alrA was leathal.

To determine if these mutations were specifically perturbing the uptake of D-alanine, a second screen was developed using a strain where the alrA null mutation was complemented by a heterologous alanine racemase gene (alrA from $B$. subtilis W23 including its own promoter) cloned into the unstable plasmid, pLOSS* (35). This strain, KS21, was chromosomally deleted for alrA but was able to grow on normal media in the absence of exogenous D-ala, due to the racemase expressed from the alr $A^{\text {W23 }}$ gene carried by the plasmid. The plasmid (pLOSS* alrA ${ }^{w 23}$ ) also constitutively expressed $\beta$-galactosidase so strains bearing the plasmid gave rise to blue colonies on media supplemented with X-gal (Fig 4A). However, pLOSS* is an unstable plasmid and can be easily lost if antibiotics selection for the plasmid is not maintained and expression of $a l r A^{w 23}$ is not essential for viability. Therefore, when the media was supplemented with D-alanine the strain was able to grow with or without the plasmid, consequently white colonies would appear among blue colonies (Fig.4 B and C). But, if a mutation that interfered with D-ala uptake was present in this strain, the plasmid-borne alrA ${ }^{\text {W23 }}$ would become essential for viability 
and hence only cells that carried the plasmid could grow and form blue colonies even in the presence of excess exogenous D-ala.

The 3 mutations initially identified as potentially being synthetically lethal with alrA null were then introduced into the modified host strain (KS21). Transformants with both null alleles were easily obtained in all cases. However, of the 3 mutations only the deletion of $y \operatorname{tnA}$ required the presence of pLOSS*alrA ${ }^{\text {W23 }}$ plasmid when grown in the presence of D-alanine (Fig. 4B and C). The other 2 null mutations ( $y f k T$ and $y d g F$ ) generated strains that easily lost the plasmid when D-ala was present in the culture medium, resulting in a high proportion of white colonies in the absence of spectinomycin (the resistance marker encoded on pLOSS*alrA $\left.{ }^{W 23}\right)$. This indicated that these strains were able to assimilate D-ala sufficiently to allow growth without the need for the complementing copy of alrA on the plasmid. Thus these genetic screens identified the transporter-like protein YntA as the possible D-alanine transporter, as the loss of $y n t A$ resulted in a strain that was not able to exploit D-alanine in the culture medium for cell growth in the absence of the alanine racemase, AlrA.

\section{Cross-feeding assay}

If YtnA was involved in the assimilation of exogenous D-ala, it would be expected that a strain lacking this transporter would not be able to re-assimilate D-ala released from the cell wall during growth. To test this hypothesis we employed a simple bioassay using the growth of the alrA null mutant as a reporter for the presence of $D$-alanine. Previous publications had suggested that $B$. subtilis does not release $D$-alanine into the culture medium during growth (1) on the basis that it was not detectable in culture supernatants. Thus the growth of a wild-type Bacillus strain was not expected to release D-ala and so would not support the growth of a D-ala auxotroph. Indeed, by streaking the alrA null mutant as a parallel line very close to a linear streak of the wild type strain (168CA) on a nutrient agar plate did not result in any growth of the alrA null mutant (Fig. 4D). In contrast, using the same inoculation technique 
using a ytnA null strain in place of 168CA resulted in clear growth of the alrA null strain (Fig. 4D), showing that the growth of the $y \operatorname{tn} A$ null strain resulted in the release of $\mathrm{D}$-ala into the surrounding medium which could be utilised by the alrA null strain. In other words, D-alanine was diffusing from the ytnA null strain in sufficient quantity to support the growth of the D-alanine auxotroph (alrA null). This released $\mathrm{D}$-alanine presumably originated from either the maturation of the peptidoglycan, through the action of DacA and LdcB (15), or the result of spontaneous cleavage of D-ala from the teichoic acids present in the cell envelope. Using the same cross-feeding assay, but with a strain deleted for both $y \operatorname{tn} A$ and the dItABCD operon (strain KS36) as the feeding strain where D-alanine is not present on the teichoic acid (3), a similar but less dramatic result was obtained (Fig. 4E), suggesting that a large proportion of released D-alanine originates from the teichoic acids under these conditions.

These results supported the idea that the putative transporter YtnA was required for D-ala uptake. We therefore rename YtnA as DatA (므-alanine transporter $\underline{A})$. The above results also show that the most likely sources of exogenous D-alanine in vegetative growth are from the teichoic acids and the maturation of the cell wall. It also demonstrated that the wild-type strain efficiently assimilated the released D-alanine, presumably for reuse in future cell wall synthesis.

\section{Detection of alanine in liquid cultures}

Since the D-alanine released by the datA null strain when grown on agar plates was sufficient to support the growth of the D-alanine auxotroph, it was expected that in liquid growth the released D-ala would be detectable at the chemical level. For this parallel cultures of 168CA and KS22 (datA null) were grown in LB medium and the culture supernatants were derivatised with Marfeys reagent prior to HPLC analysis (36) to permit separation and quantitation of optical isomers of alanine. Using a dilution series of D-ala in sterile LB medium as standards it was possible to identify the peak corresponding to D-ala on the HPLC trace (Fig S1) and generate a standard curve for the quantitative determination of D-ala in the 
sample. Using this standard curve we could show that D-ala was present in the culture medium of $B$. subtilis during exponential growth (Fig S2A and B). As expected, for the wild type strain the concentration was low, and reduced to undetectable levels at the end of exponential growth. In contrast, the datA null strain exhibited elevated levels of D-ala throughout exponential growth (Fig. S2B). A similar result was obtained for culture supernatants of the dltABCD null strains, in which Dalanine was not present on the teichoic acids (Fig. S2C), although the amount of released D-alanine was less, representing only that derived from the peptidoglycan. These results were consistent with the cross feeding results on plates.

To further clarify the role of DatA in alanine uptake/release the wild-type and the $\operatorname{datA}$ null strain were grown in a defined minimal media supplemented with either $0.5 \mathrm{mM}$ L-alanine, $\mathrm{D}$-alanine or in the absence of any alanine. The growth of the culture was then monitored and samples were taken at regular intervals to allow the concentration of free alanine in the culture medium to be determined using derivatisation with OPA and HPLC analysis (see methods). The results obtained were then plotted against time and are shown Fig 5 (which shows a characteristic experiment). When the strains were grown in the absence of alanine the HPLC analysis clearly showed a peak corresponding to alanine appearing after about 90 min of growth. However, using OPA labelling technique the isomeric form of alanine cannot be determined. As with the LB medium (Fig S2A and B), for the wild-type strain this peak was relatively small, corresponding to about 5-7 $\mu \mathrm{M}$ and was seen to disappear toward the end of exponential growth (Fig. 5A). In dramatic contrast a significantly stronger alanine peak was obtained for the $\operatorname{dat} A$ null strain starting at about the same time, but rapidly increasing to over $100 \mathrm{mM}$ and remaining at this level. Where alanine was present in the culture medium it was clear that the wild-type strain efficiently assimilated this amino acid in both isomeric forms over the duration of the experiment (Fig 5B), reducing its concentration to undetectable levels between 120 and 150 min of incubation, whereas the datA null strain was unable to efficiently utilise either isomeric form of alanine. 
Interestingly, we reproducibly observed that the wild-type strain more efficiently utilised the D-isomeric form of alanine. In contrast, the poor uptake of alanine exhibited by the datA mutant was more evident for L-alanine than for the D-isomer. We also found that, of the other amino acids tested that exhibited depletion over time in the culture medium of the wild-type strain, only alanine was reproducibly and significantly changed by the deletion of datA (data not shown).

From the chemical analysis and the cross feeding experiments, it is clear that alanine is released as $B$. subtilis grows, and strains lacking DatA do not seem as efficient as the wild type strain in the reassimilation of this amino acid. It was however evident that the concentration of alanine reduced at the end of exponential growth (Fig. 5B), suggesting that there may be other uptake systems acting to assimilate alanine in later phases of growth. However, it would seem that these are not sufficient to support vegetative growth as indicated by the synthetic lethality of $\operatorname{dat} A$ and alrA mutations.

\section{Genetic context and expression of datA}

The published transcriptional data (37) suggested that datA was the last gene in an operon with transcription starting upstream of metK and passing through asnB before reaching $\operatorname{dat} A$. To confirm this we designed a large set of oligonucleotides that would permit the amplification of various regions surrounding $\operatorname{dat} A$ using RtPCR (Fig S3B). The functionality of these primer pairs were first confirmed using genomic DNA extracted from 168CA as a template (Fig S3C). These oligonucleotide combinations were then used to generate cDNA from total RNA extracted from exponentially growing 168CA (Fig S3D). The resulting DNA fragments were then resolved on an agarose gel to provide an indication of the size of the possible RNA transcripts in this region of the genome. This analysis supports the previous microarray data (37) suggesting that datA is co-transcribed with asnB and metK, both of which have been shown to be required for growth ((38) and (39) respectively). 
Although $\operatorname{dat} A$ is the last gene in the operon, we thought it necessary to exclude the possibility that the phenotypic effects we observed for the insertional deletion of $\operatorname{dat} A$ could be due to some unforeseen effects on the expression of $a s n B$ and/or metK. For this we tested whether the phenotype could be fully complemented by ectopically expressed datA. Initially this was tested by introducing into the alrA null mutant (KS27) a copy of $\operatorname{datA}$, using its own ribosome binding site (RBS) under the control of the IPTG-inducible $\mathrm{P}_{\text {spac }}$ promoter into the amyE locus. The resulting strain then transformed with the datA null mutation using chromosomal DNA from strain BKE30530 ( $\triangle$ datA::erm). However, none of the resulting colonies carried the correct markers, suggesting that the construct at amyE was not functioning. Examining the putative RBS of datA raised the possibility that translation of the ectopic copy of $\operatorname{dat} A$ was poor. Thus, the inducible construct was reconstructed, this time changing the RBS to one closer to the consensus. A strain carrying this new construction as well as the datA deletion (KS41) was found to be easily transformed with the alrA null mutation in the presence of the inducer $(0.1 \mathrm{mM}$ IPTG; Fig S3A). Thus, enhancing the translation of the datA transcript was sufficient to complement the chromosomal null mutation, showing that the observed phenotype was caused solely by the deletion of $\operatorname{dat} A$ and not due to negative effects on the genes upstream, and that efficient expression of native $\operatorname{dat} A$ seems to be coupled with the translation of the upstream genes.

\section{Characterisation of an L-alanine auxotroph}

As mentioned above, we found that deletion of the putative alanine dehydrogenase gene, alaT, led to a severe growth defect on glucose minimal media without alanine (Fig. 6A). The defect was corrected by the addition of either D- or L-alanine in the growth medium (Fig. 6A and B), suggesting that AlaT played a major role in the biosynthesis of alanine. When incubated for an extended time this null mutant was able to generate visible biomass in the absence of alanine (Fig. $6 \mathrm{~A}$; top right quadrant), suggesting the existence of another, inefficient biosynthetic pathway. This result is consistent with an unpublished 
observation by Sonenshine and Belitsky (20), that deletion of alaT caused severe, but not complete alanine auxotrophy. It was possible that some other transaminases were able to catalyse the necessary conversion of precursors into alanine, as has been demonstrated for E. coli (40). However, as the growth of the strain was very slow in the absence of alanine (Fig. $6 \mathrm{~A}$ ), we thought it also possible that Dat, the putative aminotransferase that we identified as the "secondary" D-ala synthetic pathway, has generated limited amount of D-ala which was then converted to L-ala by the racemase, to permit limited growth (Fig. 6A). To test this we constructed a strain deleted for both alaT and dat (strain MC4). Indeed, MC4 was unable to grow on glucose minimal plates without alanine. These results show that AlaT is the main enzyme for L-alanine synthesis while dat has a minor role. Interestingly, although expression of dat has been shown to be upregulated in response to nitrogen starvation (41), we found no evidence of Dat being involved in the utilisation of D-or L-alanine as a carbon or ammonium source. Instead, this role was specifically fulfilled by the alanine dehydrogenase encoded by ald (Fig. S4) as had previously been suggested (42).

\section{DatA can transport both $\mathrm{D}$ and L-alanine}

Our phenotypic characterisation of the $\operatorname{datA}$ null mutant clearly identified DatA as the transporter of Dala. However, in the initial analysis of the alrA null strain we observed that excess L-ala in the culture medium interfered with the strain's ability to grow. An alrA dat double mutant that absolutely depended upon exogenous D-alanine under all growth conditions tested exhibited even higher sensitivity to the level of L-alanine. This suggested that L-alanine competed with D-alanine for the transporter, raising the possibility that DatA could also transport L-alanine and was not specific for the D-isomer. This possibility was further supported by the fact that both $D$ - and L-alanine assimilation was perturbed in the datA null strain (Fig. 5B). But as the strains used in these analyses were still capable of alanine biosynthesis, alanine uptake was not necessary for growth, and so might not represent the 
uptake capability of an alanine auxotroph. With AlaT being identified as the major biosynthetic enzyme in the alanine pathway, it was possible to determine if DatA was also required for L-alanine uptake. We constructed a strain deleted for both alaT and $\operatorname{dat} A$, and then tried to propagate the strain in/on GM media supplemented with L-alanine. Initial results suggested that DatA was not required for L-alanine uptake as the double mutant appeared to grow normally on minimal media supplemented with $1 \mathrm{mM} \mathrm{L-}$ alanine (Fig.6B, upper plates). However, on plates where L-alanine was provided at a lower concentration $(0.4 \mathrm{mM})$ the double mutant failed to show any growth (Fig 6B, lower plates). To confirm these observations in a more quantitative manner we analysed the growth of the strain in liquid culture using a range of alanine concentrations (Fig 6C). From the growth curves generated it was clear that normal growth of an alanine auxotroph required the presence of at least $0.8 \mathrm{mM}$ alanine in the culture medium (Fig. 6C (i)), whereas the alaT datA double mutant required at least $1 \mathrm{mM}$ to exhibit the same growth profile (Fig. 6C (ii)). Similar results were also obtained when D-alanine was used to feed the strain (Fig. 6 (iv) and (v)). Surprisingly, D-ala at concentrations over $1 \mathrm{mM}$ were able to support the growth of an alaT alanine auxotroph even when datA was deleted, suggesting that both $\mathrm{D}$ and L-alanine were able to be taken up by some other transport systems, but only when present in high concentrations.

\section{Summary:}

D-alanine is an essential component of all bacterial cell walls but it is unclear what happens to old wall as the cell grows. For Gram-negative bacteria, it is logical that the degraded material is contained within the space between the outer and inner membranes, and is available for recycling. But for Gram-positive bacteria it has been assumed that the old wall is lost to the extracellular environment. This work indicates that for $D$-alanine there is an efficient system for recycling of this amino acid in $B$. subtilis and that this system is probably conserved in other Bacillus spp. Our work also suggests a link 
between extracellular cell wall maturation and intracellular metabolism in a way that maximises the use of metabolites that would otherwise be lost to the environment, comparable to the recovery of cell wall sugars $(43,44)$. The results also provide an insight into the complexity of cellular metabolism, in terms of how biosynthetic pathways may be interlinked in such a way as to compensate for deficiencies in specific precursors. This analysis initially focused specifically on the uptake of a specific, non-canonical amino acid, D-alanine and exploited the predicted synthetic lethality expected for a D-alanine auxotroph that lost the ability to assimilate exogenous D-alanine. We identified a potential permease gene, ytnA, as a transporter gene that is necessary for efficient D-alanine uptake. Consequently, we have changed the name of $y \operatorname{tn} A$ to $\underline{\mathbf{D}}$-alanine transporter $\mathbf{A}, \operatorname{dat} A$, to reflect its apparent function in the recycle of the D-alanine that is released during the normal processes of cell wall synthesis and maturation. This role seems consistent with growth in a nutrient limited environment. Genes with significant similarity to $\operatorname{dat} A$ can be identified in most Bacilli (not shown), although none have been characterised. Interestingly, S. aureus is known to release D-alanine at the end of exponential growth, at least in laboratory culture (1), suggesting that $S$. aureus does not possess this recycling function. However, genes encoding homologues of DatA can be tentatively identified by similarity, but since the similarity is low (below $48 \%$ identity), they may have other biological roles.

Characterisation of the phenotype of the D-alanine auxotroph shows that the uptake of exogenous D-alanine is not sufficient to support high growth rates seen for a wild-type strain even in the presence of excess D-alanine (Fig. 1), indicating that the uptake is the rate limiting step. Unfortunately attempts to biochemically characterise DatA were not successful as we were unable to purify the protein. Thus we employed phenotypic and genetic methods to try and determine the specificity of the transporter. To make this possible we defined the late biosynthetic steps of L-alanine synthesis in $B$. subtilis and found that, in contrast to E. coli where a functionally redundant set of 3 transaminases (AvtA, YfbQ and SerC; (40)) participate in alanine synthesis, in B. subtilis alanine synthesis seems to be primarily 
the role of the transaminase, AlaT. Surprisingly, in our analysis of the D- and L- alanine dependent strains (alrA and alaT mutants), a second synthetic pathway for alanine was identified involving the Dalanine transaminase, Dat, though this only supported limited growth in both auxotrophs. The availability of an alanine auxotroph then allowed us to show that DatA is required for efficient uptake of exogenous L-alanine only when the amino acid is present at concentrations below $1 \mathrm{mM}$. This indicates that L-alanine uptake can occur through other non-specific transporters, but these have low affinity and so do not function well when the substrate is present a low concentrations. However, since this is an in vivo assay other factors may also be influencing uptake, such a growth of the strain that we cannot take into account.

In the light of these results we were prompted to re-evaluate alanine metabolism in B. subtilis. Previous analysis of other enzymes acting in alanine metabolism is rather limited, with two enzymes being identified in the literature from various Bacillus species. The first being an alanine dehydrogenase (Ald (42)) that had previous been identified as functioning in the use of alanine as a carbon source in sporulation; the second a D-alanine transaminase (Dat (45)) proposed to function in the metabolic conversion of D-alanine into D-glutamate (46), perhaps in stationary phase growth or during sporulation. However, transcriptional micro-array data and proteomics for both of these genes seems to suggest that these enzymes are maintained at a relatively constant abundance in cells (41), inferring more of a central role in cellular metabolism. Our analysis confirms that Ald is required for alanine to be used as a carbon source (Fig. S4), with the suggestion that this enzyme acts on only the L-isomer and Dat is implicated in the synthesis of both D- and L-alanine, either directly or in combination with the racemase (AlrA). Interestingly, the activity of Dat is inefficient in B. subtilis and is unable to fully correct the auxotrophy caused by the loss of AlaT (the main L-alanine synthase) or AlrA (the alanine racemase for D-alanine production). It was also evident that exogenous L-alanine inhibits growth of a strain lacking the alanine racemase, suggesting that the activity of Dat is modulated in some way by the availability of 
the L-isomer of alanine. These results together indicate that alanine biosynthesis could be integrated with the L-/D- glutamate biosynthetic pathway via Dat in such a way as to permit the generation of Dalanine, which in turn can be converted into the L-isomeric form for protein. This integration of pathways seems to be supported by the characterisation of other bacterial species, where it has been shown that Listeria possess an equivalent Dat enzyme which seems to be fully capable of supplying the cells D-alanine in the absence of a functional alanine racemase (32), though the mutant strains do not exhibit L-alanine sensitivity. This idea is further supported by the characterisation of an enzyme comparable to Dat in Mycobacterium smegmatis showing that it can supply the cell with D-glutamate in the absence of the cognate racemase, Murl, as well as provide D-alanine in a strain deleted for the alanine racemase (33). Thus, it would seem this set of enzymes are well conserved in a diverse range of bacteria, but most obviously in Firmicutes and close relatives. However, their activity must be modulated in different ways, presumably reflecting different metabolic priorities. This then raises the question of how the activity of these enzymes are modulated in the cytosol to ensure that the necessary precursors are available and that the metabolic pathways do not undergo futile cycles of interconversion. Our work is now focused on the subcellular localisation of these enzymes in combination with determining biochemical activity and substrate preference.

\section{General Material and methods}

Escherichia coli $\mathrm{DH} 5 \alpha$ was used for plasmids amplification. Bacillus subtilis strains were mutant derivatives of $168 \mathrm{CA}$ and their relevant characteristics are shown in Table 1. All the chemicals and reagents used in this work were obtained from Sigma except where stated otherwise.

Plasmid DNA was routinely extracted using Plasmid MiniPrep Kit (Qiagen), whilst the extraction of $B$. subtilis chromosomal DNA was either done using DNA wizard kit (Promega) for sequencing and PCR, or as a simple DNA extract (47) to permit strain construction. The transformation of $B$. subtilis 
strains was carried out according to the method developed by Anagnostopoulos and Spizizen (48), modified by Young and Spizizen (49) and E. coli transformation was done as described by Hanahan,1985 (50). Selection for antibiotic resistance markers was done using the following concentrations: chloramphenicol $(5 \mu \mathrm{g} / \mathrm{ml})$, erythromycin $(1 \mu \mathrm{g} / \mathrm{ml})$, spectinomycin and ampicillin $(100 \mu \mathrm{g} / \mathrm{ml})$ in Nutrient Agar (Oxoid). Where required 5-bromo-4-chloro-3-indolyl- $\beta$-D-galactopyranoside (X-gal) was added to a final concentration of $0.002 \%$ to provide a visual indication of $\beta$-galactosidase activity, and where required IPTG was added to a final concentration of $1 \mathrm{mM}$.

\section{Bacterial strains and culture manipulation}

The bacterial strains were routinely grown in LB (Lysogeny broth) and on nutrient agar with added supplements when required ( $0.5 \mathrm{mM}$ D-alanine; $1 \mathrm{mM}$ IPTG). Where bacterial strains were grown in a defined media based on Spizizen minimal medium (SMM, Anagnostopoulos and Spizizen (48), Young and Spizizen (49); $0.2 \% \mathrm{w} / \mathrm{v}$ ammonium sulphate, $1.4 \%$ dipotassium phosphate, $0.6 \%$ potassium dihydrogen phosphate, $0.1 \%$ sodium citrate dehydrate, $0.02 \%$ magnesium sulphate, to which was added $5 \mathrm{mM}$ magnesium sulphate heptahydrate, $0.1 \mathrm{mM}$ calcium chloride dehydrate, $65 \mu \mathrm{M}$ manganese (II) sulphate tetrahydrate) this provided a basic salts medium to which supplements were added to permit growth (where necessary glucose was added to a final concentration of $10 \mathrm{~g} / \mathrm{l}$ and each amino acids were present at a final concentration of $1.25 \mathrm{mM}$ each except where specifically stated). However where amino acid utilisation as a nitrogen source was investigated the SMM was modified by omitting the ammonium sulphate. To simplify the description of the media we have adopted $\mathrm{M}$ to denote minimal with any omissions in brackets followed by the additions, $G$ for glucose, A for amino acids or the three letter code for individual amino acids, again indicating any omitted components in brackets. Hence, MGA indicates the medium with salts, glucose and all amino acids added, while M-NH3 G D-Ala indicates that the minimal medium has the salts, but lacks the ammonium sulphate, Glucose and Dalanine. 
For growth curve assays in LB, the bacterial strains of interest were grown in LB or defined media (supplemented with $\sim 450 \mu \mathrm{M}$ of D-alanine where necessary) at $30^{\circ} \mathrm{C}$ overnight. The overnight cultures were diluted 1:30 with pre-warmed medium and grown for about 2 hours at $37^{\circ} \mathrm{C}$ to obtain exponentially growing cultures. The optical density of the culture were determined $\left(\mathrm{OD}_{600 \mathrm{~nm}}\right)$ and measured volumes of the cultures were centrifuged for 3 minutes in a benchtop centrifuge (RCF 9,509). The cell pellets were quickly suspended in pre-warmed culture medium and dispensed into pre-warmed flasks or the wells of microplates, which were already loaded with pre-warmed medium such that the final $\mathrm{OD}_{600}$ in each flask was $\sim 0.1$ in each flask and $\sim 0.05$ in each well of microplate. The cultures were then incubated with shaking and the optical density (OD $\left.{ }_{600 \mathrm{~nm}}\right)$ measured at regular intervals using a spectrophotometer or automatically by the plate reader (BMG Fluorostar). Where the experiment required the medium composition to be changed cultures were grown overnight in a complete medium and back diluted as described above into the same medium and incubated for $2 \mathrm{~h}$. The optical density at $600 \mathrm{~nm}\left(\mathrm{OD}_{600}\right)$ was the determine and measured volumes of the cultures were centrifuged at 13,300 rpm for 3 minutes in a benchtop centrifuge (Heraeus Pico17, 45 angle rotor, 24 places, RCF $17000 \times \mathrm{g}$ ). The pellets were suspended in appropriate volumes of the new medium and distributed into culture vessels, either Flasks or sterile microplates that were pre-loaded with appropriate volume of media with relevant supplements such that the correct final optical density was obtained. The growth of the strains was then monitored as described above.

\section{Determination of growth on solid media}

For growth analysis using solid media, agar plates $(1.5 \% \mathrm{w} / \mathrm{v})$ were prepared using SMM salts to which was added $5 \mathrm{mM}$ magnesium sulphate, $0.1 \mathrm{mM}$ calcium chloride dehydrate and $65 \mu \mathrm{M}$ manganese (II) sulphate. Variations of this base medium were made by the addition of different supplements: in general $10 \mathrm{~g} / \mathrm{l}$ glucose was added as a carbon source (Glucose minimal; GM), plates supplemented with $98 \mu \mathrm{M}$ L-tryptophan (GMT) or 1 mM L-alanine; GMTala and GMala plates were supplemented with L- 
tryptophan and $1 \mathrm{mM}$ D-alanine or alanine alone, GMaa plates contained $1.25 \mathrm{mM}$ of the 19 standard amino acids omitting alanine. For plates testing for utilisation of alanine as sole carbon source, glucose was removed from SMM and replaced with $25 \mathrm{mM} \mathrm{L-} \mathrm{or} \mathrm{D-alanine} \mathrm{(Mala).} \mathrm{For} \mathrm{those} \mathrm{testing} \mathrm{for}$ utilisation of alanine as a sole nitrogen source, ammonium sulphate were removed from SMM salt mix and the medium supplemented with $10 \mathrm{~g} / \mathrm{I}$ glucose and $25 \mathrm{mM}$ alanine (GM-NH 3 ala plates). Strains were streaked on prepared agar plates and incubated at $37^{\circ} \mathrm{C}$ for 24 hours (GMaa plates) or 48 hours (GMT, GM, GMala, Mala and GM-NH 3 ala plates) and growth determined by visual inspection was recorded by photography.

\section{Bacterial strain construction}

Mutants with single gene deletion were obtained from the Bacillus Genetic Stock Centre (BGSC) where single gene deletions had been generated by insertional deletion using a erythromycin resistance cassette (51). Chromosomal DNA from these strains at low DNA concentrations were used to transform the wild-type strains $168 \mathrm{CA}$ or $168+$ to ensure isogenic strains were characterised. Where necessary the temperature-sensitive vector pDR244 (51) with a functional cre recombinase gene was used to loop out the erythromycin marker between the lox sites, leaving the resultant strain a marker-less deletion mutant. Repetition of this method was used to generate double or triple mutants where required. The transformed strains were checked that they had the appropriate antibiotic resistance, and the gene knockouts were confirmed by PCR. The list of constructed strains of $B$. subtilis is shown in Table 1.

To identify potential D-alanine synthesis pathways, searches of the bioinformatics databases (SubtiWiki, SubCyc and Metacyc) were used to identify any genes that have either putative or been functionally characterised to have roles in D-amino acid metabolism. Strains deleted for the genes identified were obtained from BGSC and are listed in Table S4.

The deletion of the dlt operon was generated by ligation of 3 DNA fragments generated by PCR. Firstly, oligonucleotides oKSO1 and oKSO2 were used to amplify a $2.0 \mathrm{~kb}$ DNA fragment, 
complementary to the DNA sequence upstream of the dItA gene. Then oKS03 and oKSO4 were used to generate a $2.0 \mathrm{~kb}$ DNA fragment at the downstream of the dItD gene. Both of these DNA fragments were then digested with $\mathrm{Bg} / \mathrm{Il}$, and after purification, appropriate quantities of the digested DNA fragments were ligated with a chloramphenicol resistant cassette (cat) obtained from plasmid pSG1 as a BamHI fragment. The ligation product was used directly in the transformation of $B$. subtilis $168 C A$ strain. The correct knockout strain ( $\triangle$ dltABCD::cat; KS11) was isolated as a chloramphenicol resistant transformant and confirmed by PCR.

\section{Amino acid competition assay}

The wild-type strain (168CA) and the D-alanine dependent strain RD 180 ( $\Delta a$ lrA::zeo) were grown in LB medium, supplemented with $450 \mu \mathrm{M}$ of D-alanine to which other individual amino acids (Melford Bioscience) at concentrations between $0.0098 \mathrm{mM}-5.0 \mathrm{mM}$ were added. The growth of the strains at $37^{\circ} \mathrm{C}$ was then monitored over time using microplate reader (BMG, Fluorostar). The readings obtained were then used to plot growth curves for the culture to determine if the addition of a specific amino acid had any specific significant impact on D-alanine dependent strain (RD180) and not on the wild-type strain (168CA).

\section{Synthetic lethal screen}

To determine if a specific transporter was required for the uptake of D-alanine by the D-alanine auxotroph, (strain RD180) the auxotroph was systematically transformed with insertional mutations in genes potentially encoding amino acid transporter proteins. Using the premise that the loss of this transporter in the alrA null background would be lethal and very few colonies bearing the incoming resistance marker would be obtained compared to other null mutations. Our initial screen utilised the pMUTIN knockout collection (obtained from the NBRP strain collection, Japan (52)) as a source of null mutations and a list of the mutations tested is shown in Table S4. Where a significant reduction in 
transformation efficiency was seen, the gDNA was used a second time transforming both the alrA null strain and the wild-type strain in parallel to confirm that it was the genetic background and not the quality of the DNA preparation causing the reduced transformation efficiency. Where reduced transformation was confirmed, the resulting colonies were then screened for the presence of expected resistance markers for the respective mutations (zeocine for alrA, and erythromycin for pMUTIN,).

\section{Stabilisation of pLOSS*alrA by deletion of datA}

Plasmid pLOSS* alrA ${ }^{w 23}$ was constructed by cloning a PCR generated fragment of DNA corresponding to the alrA gene encoded by B. subtilis W23 into the pLOSS* vector described by Claessen et al. (35), inserting it such that it was under the control of the $\mathrm{P}_{\text {spac }}$ promoter (using $\mathrm{BamHI}$ and $\mathrm{Xbal}$ sites introduced by the PCR oligonucleotides alrAW23-1 and alrAW23-2). Strain RD180 (AalrA::zeo) was then transformed with pLOSS * alrA ${ }^{w 23}$ to give strain KS21 ( $\triangle$ alrA pLOSS* alrA ${ }^{w 23}$ ), which exhibited plasmid instability when grown in the presence of D-alanine (see results).

Strain KS21 was then transformed with gDNA from BKE30530 ( $\triangle$ datA::erm) selecting for erythromycin resistance to produce strain $\mathrm{KS} 23$ strain $\left(\triangle\right.$ datA $\triangle$ alrA pLOSS* alrA $\left.{ }^{W 23}\right)$. These two were grown on plates of nutrient agar supplemented with X-gal and with and without $\mathrm{D}$-alanine at $37^{\circ} \mathrm{C}$ to determine the stability of the plasmid as indicated by the loss of $\beta$-galactosidase activity.

\section{Complementation of datA.}

The coding sequence of the $\operatorname{dat} A$ gene and 30 bases upstream, to include the putative ribosome binding site (RBS) of the gene, was inserted into plasmid pPY18 (bla amyE3' cat $P_{\text {spac }}$ lacl amyE5';(53)) to produce plasmid pKS1 (bla amyE3' cat $P_{\text {spac }}$ datA lacl amyE5'). Plasmid pKS6 was similarly constructed, but in this case the native ribosome binding of $\operatorname{dat} A$ was modified (to a sequence corresponding to the RBS identified upstream of $f t s L)$. Both pKS1 and pKS6 were then used to transform strain KS22 ( $\triangle$ datA::erm) such that the amyE locus on the chromosome was replaced by the inducible copy of datA to give strains, KS26 and KS41. Correct replacements were confirmed by determining the loss of amylase activity in the 
strain and by PCR. Both of these strains were then transformed with the gDNA from RD180 strain $(\triangle a l r A:: z e o)$ selecting for zeocine resistance in the presence of $0.5 \mathrm{mM} \mathrm{D}$-alanine and $1.0 \mathrm{mM}$ IPTG to give strains KS27 and KS42.

\section{Cross-feeding assay}

In this assay the D-alanine auxotroph strain (RD180) was grown as close as possible to wild type or test strains on a solid medium, ensuring that the strains did not become mixed. Through trial and error it was determined that the D-alanine prototroph (donor; $a / r A^{+}$) had to be inoculated onto the plate first and incubated for $3.0 \mathrm{~h}$ at $37^{\circ} \mathrm{C}$ prior to inoculating the D-alanine auxotroph (recipient) strain in parallel to the donor strain. The plate was then incubated for a further $23 \mathrm{~h}$ and the resulting growth photographed.

\section{Culture sample processing for RP-HPLC analysis for D-alanine}

Samples were taken from the culture at different time points were centrifuged at $3000 \times \mathrm{g}$ for $5.0 \mathrm{~min}$ and the culture supernatant was filtered through sterile syringe filter (pore size $0.2 \mu \mathrm{m}, \mathrm{GILSON}^{\circ}$ ). The resulting filtrate was then passed through a spin column (Vivaspin 2.0 Hydrosart, 2000 MWCO, Generon Ltd.) before analysis. For standards, the LB medium sample was similarly processed with and without added known concentrations of D-alanine. A $150 \mu \mathrm{l}$ of the "super" filtered culture supernatant was concentrated to approximately $50 \mu$ using a SpeedVac concentrator (SavantSPD131DDA, Thermo Electron Corporation). To this material $150 \mu$ l of Marfey's reagent (1.0 \% Na-(2,4-Dinitro-5fluorophenyl)-L-alaninamide in acetone) was added and mixed. Then, $40 \mu$ l of sodium bicarbonate (1.0 M) was added and mixed prior to incubation for $1.0 \mathrm{~h}$ at $40 \mathrm{C}^{\circ}$ with shaking at $750 \mathrm{rpm}$ (Thermomixer compact, Eppendorf). The mixture was then allowed to cool and $25 \mu \mathrm{l}$ of $\mathrm{HCl}(2.2 \mathrm{M})$ was added to stop the reaction and dried by SpeedVac before being suspended in $150 \mu$ l of HPLC suspension solution (90\% of $0.05 \mathrm{M}$ triethylamaine phosphate $(\mathrm{pH} 3.0)$ and $10 \%$ of acetonitile $+0.1 \%$ formic acid). The resulting suspension was then filtered through $0.22 \mu \mathrm{m}$ centrifuge tube filter (cellulose acetate filter, Costar) at 
full speed in a micro centrifuge for $1.0 \mathrm{~min}$, and the filtrate was use for HPLC analysis. Results shown in

Fig 5 are representative for 3 independent experiments.

Samples labelled by Marfeys reagent (36) were analysed using a Perkin Elmer series 200 HPLC

with a Spheri-5, RP-18 column, dimension $100 \mathrm{~mm} \times 4.6 \mathrm{~mm}$ and particle size $5.0 \mu \mathrm{m}$ (Perkin Elmer) and a column guard, NewGuard RP-18, $7.0 \mu \mathrm{m}, 15 \times 3.2 \mathrm{~mm}$ (Perkin Elmer). The mobile phase was generated from $0.05 \mathrm{M}$ triethylamine phosphate $(\mathrm{pH} 3.0)$ (solution $\mathrm{A}$ ) and acetonitrile $+0.1 \%$ formic acid (solution B) as a linear gradient of solution B from $10 \%$ to $25 \%$ in $40 \mathrm{~min}$. The flow rate was $1.0 \mathrm{ml} / \mathrm{min}$ at $35^{\circ} \mathrm{C}$ and the detection wave length was $340 \mathrm{~nm}$, using a S200 Diode array detector (DAD). After each run, the column was washed with water (solution D) and then with $60 \%$ methanol (solution C). The details of the run parameters are shown in (Table S3).

\section{Determination of amino acid changes in culture media.}

To identify what effect the datA mutation had on the assimilation/release of alanine in a growing culture, parallel cultures of the wild-type (168) and the datA null strain (KS22) were grown overnight at $30{ }^{\circ} \mathrm{C}$ in a defined minimal medium; GM Opt (M. Chow, personal communication) which was GM media supplemented with amino acids serine, glutamine, asparagine and glutamate at $0.5 \mathrm{mM}$ and all others at $0.25 \mathrm{mM}$ except alanine which was omitted. The cultures were then diluted $1 / 50$ in fresh media and incubated at 37 degrees for $1 \mathrm{~h}$ prior to being diluted in the same medium to an optical density of 0.05 at $600 \mathrm{~nm}$. At this point the cultures were divided into 3 aliquots. The first was supplemented with Dalanine at $0.5 \mathrm{mM}$, and the second L-alanine $(0.5 \mathrm{mM})$ and the last was left without any addition. At regular intervals the culture optical density was determined and $1 \mathrm{ml}$ samples were centrifuged to obtain the culture supernatant, which was filtered through a Microcon centrifugal filter unit (MWCO 3kDa; Sigma) and stored at $-20^{\circ} \mathrm{C}$.

The frozen samples were then processed for HPLC analysis to determine the concentration of alanine present using pre-column OPA derivatisation as described by Henderson et al. (2008) (54) using 
an Agilent $1100 \mathrm{HPLC}$ with a Poroshell $120 \mathrm{C} 184 \mu \mathrm{m}, 4.6 \times 150 \mathrm{~mm}$ column. The resulting HPLC traces were then analysed with reference to a TO sample and known standard concentrations of alanine to permit the identification of the peak corresponding to alanine in the trace and to allow its concentration to be determined. The results were then plotted against time and the values obtained for a typical experiment are shown in figure 5.

\section{Acknowledgments:}

We would like to thank D. Thwaites for his technical help and advice with respect to amino acid transporters, A. Guyet and L. J. Wu for critical reading of the manuscript. This work was supported by the Kurdistan regional government-IRAQ in the form of a studentship for KS, and Engineering and Physical Sciences Research Council grant EP/N031962/1 (RD)

\section{References:}

1. Lam H, Oh DC, Cava F, Takacs CN, Clardy J, de Pedro MA, et al. D-amino acids govern stationary phase cell wall remodeling in bacteria. Science. 2009;325(5947):1552-5.

2. Hernandez SB, Cava F. Environmental roles of microbial amino acid racemases. Environmental Microbiology. 2016;18(6):1673-85.

3. Perego M, Glaser P, Minutello A, Strauch MA, Leopold K, Fischer W. Incorporation of D-alanine into lipoteichoic acid and wall teichoic acid in Bacillus subtilis. Identification of genes and regulation. The Journal of Biological Chemistry. 1995;270(26):15598-606.

4. Wecke J, Perego M, Fischer W, Perego M, Glaser P, Minutello A, et al. D-alanine deprivation of Bacillus subtilis teichoic acids is without effect on cell growth and morphology but affects the autolytic activity Incorporation of D-alanine into lipoteichoic acid and wall teichoic acid in Bacillus subtilis. Identification of genes and regulation. Microb Drug Resist. 1996;2(1):123-9.

5. Peng B, Su YB, Li H, Han Y, Guo C, Tian YM, et al. Exogenous alanine and/or glucose plus kanamycin kills antibiotic-resistant bacteria. Cell Metabolism. 2015;21(2):249-62.

6. Yasuda Y, Tochikubo K. Relation between D-glucose and L- and D-alanine in the initiation of germination of Bacillus subtilis spore. Microbiology and immunology. 1984;28(2):197-207.

7. Shrestha R, Lockless SW, Sorg JA. A Clostridium difficile alanine racemase affects spore germination and accommodates serine as a substrate. The Journal of Biological Chemistry. 2017;292(25):10735-42. 
8. McKevitt MT, Bryant KM, Shakir SM, Larabee JL, Blanke SR, Lovchik J, et al. Effects of endogenous D-alanine synthesis and autoinhibition of Bacillus anthracis germination on in vitro and in vivo infections. Infection and Immunity. 2007;75(12):5726-34.

9. Ferrari E, Henner DJ, Yang MY. Isolation of an Alanine Racemase Gene from Bacillus subtilis and its Use for Plasmid Maintenance in B. subtilis. Bio/Technology. 1985;3:1003.

10. Xia Y, Chen W, Zhao J, Tian F, Zhang H, Ding X. Construction of a new food-grade expression system for Bacillus subtilis based on theta replication plasmids and auxotrophic complementation. Applied Microbiology and Biotechnology. 2007;76(3):643-50.

11. Koch AL, Doyle RJ. Inside-to-outside growth and turnover of the wall of Gram-positive rods. Journal of theoretical biology. 1985;117(1):137-57.

12. Pooley HM. Layered distribution, according to age, within the cell wall of Bacillus subtilis. Journal of Bacteriology. 1976;125(3):1139-47.

13. Pooley HM. Turnover and spreading of old wall during surface growth of Bacillus subtilis. Journal of Bacteriology. 1976;125(3):1127-38.

14. Atrih A, Bacher G, Allmaier G, Williamson MP, Foster SJ. Analysis of peptidoglycan structure from vegetative cells of Bacillus subtilis 168 and role of PBP 5 in peptidoglycan maturation. Journal of Bacteriology. 1999;181(13):3956-66.

15. Hoyland CN, Aldridge C, Cleverley RM, Duchene MC, Minasov G, Onopriyenko O, et al. Structure of the LdcB LD-carboxypeptidase reveals the molecular basis of peptidoglycan recognition. Structure. 2014;22(7):949-60.

16. Neuhaus FC, Baddiley J. A continuum of anionic charge: structures and functions of D-alanylteichoic acids in Gram-positive bacteria. Microbiol Mol Biol Rev. 2003;67(4):686-723.

17. Radkov AD, Moe LA. Bacterial synthesis of D-amino acids. Applied Microbiology and Biotechnology. 2014;98(12):5363-74.

18. Walsh CT. Enzymes in the D-alanine branch of bacterial cell wall peptidoglycan assembly. The Journal of Biological Chemistry. 1989;264(5):2393-6.

19. Pierce KJ, Salifu SP, Tangney M. Gene cloning and characterization of a second alanine racemase from Bacillus subtilis encoded by yncD. FEMS microbiology letters. 2008;283(1):69-74.

20. Belitsky BR. Biosynthesis of Amino acids of the Glutamate and Aspartate Families, Alanine, and Polyamines. In: Sonenshein AL, editor. Bacillus subtilis and its Closest Relatives: from Genes to cells Washington, D. C.: ASM Press; 2002. p. 203-31.

21. Fotheringham IG, Bledig SA, Taylor PP. Characterization of the genes encoding D-amino acid transaminase and glutamate racemase, two D-glutamate biosynthetic enzymes of Bacillus sphaericus ATCC 10208. Journal of bacteriology. 1998;180(16):4319-23.

22. Berberich R, Kaback M, Freese E, Reese I. D-amino acids as inducers of L-alanine dehydrogenase in Bacillus subtilis. J Biol Chem. 1968;243:1006-11.

23. Freese $\mathrm{E}$, Park SW, Cashel $\mathrm{M}$. The Developmental significance of alanine dehydrogenase in Bacillus subtilis. Proceedings of the National Academy of Sciences of the United States of America. 1964;51:1164-72.

24. Siranosian KJ, Ireton K, Grossman AD. Alanine dehydrogenase (ald) is required for normal sporulation in Bacillus subtilis. Journal of Bacteriology. 1993;175(21):6789-96.

25. Stannek L, Thiele MJ, Ischebeck T, Gunka K, Hammer E, Volker U, et al. Evidence for synergistic control of glutamate biosynthesis by glutamate dehydrogenases and glutamate in Bacillus subtilis. Environmental Microbiology. 2015;17(9):3379-90.

26. Osborne SE, Tuinema BR, Mok MC, Lau PS, Bui NK, Tomljenovic-Berube AM, et al. Characterization of DalS, an ATP-binding cassette transporter for D-alanine, and its role in pathogenesis in Salmonella enterica. The Journal of Biological Chemistry. 2012;287(19):15242-50. 
27. Yu J, Ge J, Heuveling J, Schneider E, Yang M. Structural basis for substrate specificity of an amino acid ABC transporter. Proceedings of the National Academy of Sciences of the United States of America. 2015;112:5243-8.

28. M. J Zimbro, D. A. Power, S. M. Miller, G. E. Wilson, J. A. Johnson. Difco? \& BBL? Manual Manual of Microbiological Culture Media. In: Becton DaC, Sparks MU, editors. 2nd ed: BD Diagnostics Diagnostic Systems; 2009.

29. Steil L, Serrano M, Henriques AO, Volker U. Genome-wide analysis of temporally regulated and compartment-specific gene expression in sporulating cells of Bacillus subtilis. Microbiology. 2005;151(Pt 2):399-420.

30. Kimura K, Tran LS, Itoh Y. Roles and regulation of the glutamate racemase isogenes, racE and yrpC, in Bacillus subtilis. Microbiology. 2004;150(Pt 9):2911-20.

31. Miyamoto T, Katane M, Saitoh $Y$, Sekine M, Homma H. Identification and characterization of novel broad-spectrum amino acid racemases from Escherichia coli and Bacillus subtilis. Amino Acids. 2017;49(11):1885-94.

32. Thompson RJ, Bouwer HG, Portnoy DA, Frankel FR. Pathogenicity and immunogenicity of a Listeria monocytogenes strain that requires D-alanine for growth. Infection and Immunity. 1998;66(8):3552-61.

33. Mortuza R, Aung HL, Taiaroa G, Opel-Reading HK, Kleffmann T, Cook GM, et al. Overexpression of a newly identified D-amino acid transaminase in Mycobacterium smegmatis complements glutamate racemase deletion. Molecular Microbiology. 2018;107:198-213.

34. Vagner $V$, Dervyn E, Ehrlich SD. A vector for systematic gene inactivation in Bacillus subtilis. Microbiology. 1998;144 ( Pt 11):3097-104.

35. Claessen D, Emmins R, Hamoen LW, Daniel RA, Errington J, Edwards DH. Control of the cell elongation-division cycle by shuttling of PBP1 protein in Bacillus subtilis. Molecular Microbiology. 2008;68(4):1029-46.

36. Szokan G, Mezo G, Hudecz F. Application of Marfey's reagent in racemization studies of amino acids and peptides. J Chromatogr. 1988;444:115-22.

37. Nicolas P, Mader U, Dervyn E, Rochat T, Leduc A, Pigeonneau N, et al. Condition-dependent transcriptome reveals high-level regulatory architecture in Bacillus subtilis. Science. 2012;335(6072):1103-6.

38. Dajkovic A, Tesson B, Chauhan S, Courtin P, Keary R, Flores P, et al. Hydrolysis of peptidoglycan is modulated by amidation of meso-diaminopimelic acid and $\mathrm{Mg}(2+)$ in Bacillus subtilis. Molecular Microbiology. 2017;104(6):972-88.

39. Rigden DJ, Fernández XM. The 2018 Nucleic Acids Research database issue and the online molecular biology database collection. Nucleic Acids Research. 2018;46(D1):D1-D7.

40. Yoneyama H, Hori H, Lim SJ, Murata T, Ando T, Isogai E, et al. Isolation of a mutant auxotrophic for L-alanine and identification of three major aminotransferases that synthesize L-alanine in Escherichia coli. Biosci Biotechnol Biochem. 2011;75(5):930-8.

41. Tam le T, Eymann C, Antelmann H, Albrecht D, Hecker M. Global gene expression profiling of Bacillus subtilis in response to ammonium and tryptophan starvation as revealed by transcriptome and proteome analysis. Journal of molecular microbiology and biotechnology. 2007;12(1-2):121-30.

42. Siranosian KJ, Ireton K, Grossman AD. Alanine dehydrogenase (ald) is required for normal sporulation in Bacillus subtilis. Journal of Bacteriology. 1993;175:6789-96.

43. Borisova M, Gaupp R, Duckworth A, Schneider A, Dalugge D, Muhleck M, et al. Peptidoglycan Recycling in Gram-Positive Bacteria Is Crucial for Survival in Stationary Phase. mBio. 2016;7(5).

44. Reith J, Mayer C. Peptidoglycan turnover and recycling in Gram-positive bacteria. Applied microbiology and biotechnology. 2011;92(1):1-11. 
45. Taylor PP, Fotheringham IG. Nucleotide sequence of the Bacillus licheniformis ATCC 10716 dat gene and comparison of the predicted amino acid sequence with those of other bacterial species. Biochim Biophys Acta. 1997;1350(1):38-40.

46. Martinez del Pozo A, Merola M, Ueno H, Manning JM, Tanizawa K, Nishimura K, et al. Activity and spectroscopic properties of bacterial D-amino acid transaminase after multiple site-directed mutagenesis of a single tryptophan residue. Biochemistry. 1989;28(2):510-6.

47. Ward JB, Zahler SA. Genetic studies of leucine biosynthesis in Bacillus subtilis. Journal of Bacteriology. 1973;116:719-26.

48. Anagnostopoulos C, Spizizen J. Requirements for transformation in Bacillus subtilis. Journal of Bacteriology. 1961;81(5):741-6.

49. Young FE, Spizizen J. Physiological and genetic factors affecting transformation of Bacillus subtilis. Journal of Bacteriology. 1961;81:823-9.

50. D H. Techniques for transformation of E.coli. In: Glover DMIP, editor. DNA cloning - A practical approach Oxford. Washington D.C: pp. 109-135. 1985.

51. Koo BM, Kritikos G, Farelli JD, Todor H, Tong K, Kimsey H, et al. Construction and Analysis of Two Genome-Scale Deletion Libraries for Bacillus subtilis. Cell Syst. 2017;4(3):291-305.e7.

52. Kobayashi K, Ehrlich SD, Albertini A, Amati G, Andersen KK, Arnaud M, et al. Essential Bacillus subtilis genes. Proceedings of the National Academy of Sciences of the United States of America. 2003;100(8):4678-83.

53. Duggin IG, Andersen PA, Smith MT, Wilce JA, King GF, Wake RG. Site-directed mutants of RTP of Bacillus subtilis and the mechanism of replication fork arrest. J Mol Biol. 1999;286(5):1325-35.

54. Henderson JWR, R. D.; Bidlingmeyer, B. A.; Woodward, C. Rapid, Accurate, Sensitive, and Reproducible HPLC Analysis of Amino Acids. Agilent Technical note. 2008.

55. Errington J. Efficient Bacillus subtilis cloning system using bacteriophage vector phi 105J9. J Gen Microbiol. 1984;130(10):2615-28. 


\section{Figure legends:}

\section{Figure 1}

Panel A - The growth characteristics of D-alanine auxotroph (alrA) strain. The growth curves of wildtype (168CA) and $\triangle$ alrA::zeo (RD180) strains in LB medium, supplemented with different concentrations of D-alanine (0.2-1 mM) at $37^{\circ} \mathrm{C}$. The growth curves were monitored by plate reader and shown in Log10 of optical density (OD600). The plotted line shows the mean values for 3 biological replicates with error bars indicating the standard deviation between replicates.

Panel B - The growth rates of the same strains measured at three time point intervals $(60-80,80-100$ and 100-120 $\mathrm{min}$ ) of panel (A). The error bars represent the standard deviation of mean of growth rate in two independent experiments.

Panels C and D - Growth curves showing the inhibitory effect of increasing concentrations of L-alanine, glycine or L- proline on the growth of the D-alanine dependent strain (RD180) compared to the wild type strain (168CA). Both strains were grown at $37^{\circ} \mathrm{C}$ in LB medium with fixed concentration of D-alanine and supplemented with different concentrations of L-alanine (C) or $5 \mathrm{mM}$ Glycine or L-Proline (D) at $37 \mathrm{C}^{\circ}$.

\section{Figure 2}

Inhibitory effect of L-alanine on the growth of a D-alanine auxotroph in a defined media. The growth of wild-type (168CA), $\Delta a / r A(R D 180)$ and $\Delta a l r A \Delta d a t$ (KS78) in GMaa with 1 or $0.5 \mathrm{mM}$ of D-alanine or no alanine were shown in panels $\mathbf{A}, \mathbf{B}$ and $\mathbf{C}$ respectively. Strains were grown in GMaa with $1 \mathrm{mM}$ D-alanine supplemented with different concentrations of L-alanine (D-F), or in GMaa with L-alanine but without Dalanine (G-I). Growth determine in terms of optical density $(600 \mathrm{~nm})$ in plate reader with shaking at 37 ${ }^{\circ} \mathrm{C}$. Numbers in the legend represents different molarities $(\mathrm{mM})$ of either D-/L-alanine (for A-C): Dalanine; for D-I: L-alanine) in the media. '-' indicates no alanine was added in the media.

\section{Figure 3}


Identification of the second D-alanine synthetic enzyme.

Panel A - The D-alanine auxotroph strains $\Delta a l r A$ (RD180), $\Delta$ alrA $\Delta y i t F(K S 35), \Delta a l r A \Delta y r p C$ (KS34), and $\Delta a l r A \Delta \operatorname{racX}(\mathrm{KS} 33)$ were found to be able to grow well on Minimal medium (MG) in the absence of Dalanine, (plate was incubated at $37^{\circ} \mathrm{C}$ for 36 hours).

Panel B - Growth of strains $\Delta$ alrA $\Delta$ alrB (KS32), $\Delta$ alrA (RD180) and $\Delta a \operatorname{lr} A \Delta d a t$ (KS78) on MM with and without supplementation of $0.5 \mathrm{mM} \mathrm{D}$ - or L-alanine, at $37^{\circ} \mathrm{C}$ for 48 hours. As a positive control the strains were also inoculated on nutrient agar (NA) supplemented with D-alanine (0.5 mM)

\section{Figure 4}

Genetic confirmation of the role of datA in D-alanine uptake. A D-alanine dependent strain with the sole functional copy of alrA encoded on a unstable plasmid was constructed, KS21 ( $\triangle$ alrA + pLOSS* $\Omega$ alrA) and compared to a strain also lacking $\operatorname{datA}, \operatorname{KS} 23\left(\triangle a l r A \Delta d a t A+p L O S S^{*} \Omega\right.$ alrA $)$ when grown on different media. Panel A shows the strains grown on nutrient agar (NA) plus X-gal. In contrast panels B and $\mathbf{C}$ show the same strains on NA + X-gal supplemented with $450 \mu \mathrm{M}$ of D-alanine and X-gal and 900 $\mu \mathrm{M}$ of D-alanine respectively. All three plates were photographed after $16 \mathrm{~h}$ incubation at $37^{\circ} \mathrm{C}$. The blue colonies indicate that the pLOSS* $\Omega$ alrA plasmid is conserved in the cells, whereas the cells produced white colonies after losing the plasmid. (D \& E) In this cross-feeding assay, the 168CA, KS11

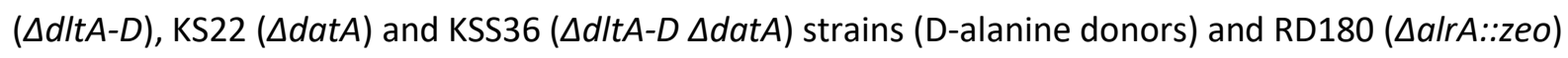
and KS12 ( $\triangle a l r A \Delta d I t A-D)$ strains (D-alanine recipients) were used in this assay. The D-alanine donor strains were firstly streaked on nutrient agar plates and incubated for $3 \mathrm{~h}$ at $37^{\circ} \mathrm{C}$. Later, the $\mathrm{D}$-alanine recipient strains were streaked closely parallel to the donor strains and kept incubating for a further 23 h. The total incubation time was $26 \mathrm{~h}$.

\section{Figure 5}


Determination the concentration of alanine present in the culture medium during growth of wild-type $B$. subtilis and a datA null strain by OPA derivatisation and HPLC. The line graphs show growth curves expressed as the optical density of the cultures at $600 \mathrm{~nm}(\bigcirc$ symbols for the wild type and $\bullet$ for the datA null strain and the column plots in panel A indicate the concentration of free alanine $(\mu \mathrm{M})$. Panel B presents the growth curves for the wild-type strain and the datA null strain when grown the presence of exogenous alanine $(0.5 \mathrm{mM})$, for simplicity the growth curves shown are for the cultures supplemented with D-alanine as those supplemented with L-alanine were essentially identical. On the same plot the alanine concentration in the respective cultures was determined over time with triangles for L-alanine ( $\triangle$ for the wild-type culture and $\boldsymbol{\Delta}$ for the $\operatorname{dat} A$ mutant) and inverted triangles for D-alanine ( $\nabla$ for the wild-type and $\nabla$ datA mutant).

\section{Figure 6}

DatA transports both isoforms of alanine. Panel A shows how the growth of $\triangle$ alaT (MC2) and $\triangle a l a T$ $\Delta$ dat (MC4) on GM plates is altered by the presence or absence of $1 \mathrm{mM} \mathrm{L-alanine.} \mathrm{The} \mathrm{plates} \mathrm{were}$ incubated at $37^{\circ} \mathrm{C}$ for 48 hours. Panel B Shows how the growth of wild-type (168+), $\Delta$ alaT (MC2) and AalaT $\triangle$ datA (MC8) on GMaa plates with either D-/L-alanine at higher (1 mM) and lower (0.4 mM) concentrations. The plates were incubated at $37^{\circ} \mathrm{C}$ for 24 hours. Panel C shows typical growth curves obtained for the wild-type strain (168), the alaT null strain (MC2) and the double mutant alaT datA (MC4, identical results were obtained for strain MC8) grown at $37^{\circ} \mathrm{C}$ in a plate reader in the liquid GMaa supplemented with a range of concentrations of D-/L-alanine from $0.4 \mathrm{mM}$ to $1 \mathrm{mM}(\mathrm{C})$. 


\section{Table 1 Strains collection}

\begin{tabular}{|c|c|c|}
\hline $\begin{array}{l}\text { B. subtilis } \\
\text { strains }\end{array}$ & Genotype & $\begin{array}{c}\text { Construction or Source or } \\
\text { Reference }\end{array}$ \\
\hline $168 \mathrm{CA}$ & trpC2 Bacillus subtilis & Laboratory strains collection \\
\hline $168+$ & Prototrophic derivative of $168 \mathrm{CA}$ & R. Daniel, unpublished \\
\hline W23 & Bacillus subtilis W23 & Laboratory strain collection \\
\hline RD180 & $\operatorname{trpC2} \Delta$ alrA::zeo & R. Daniel, unpublished \\
\hline BKE30530 & trpC2 $\triangle$ datA::erm & BGSC (51) \\
\hline BKE 17640 & $\operatorname{trp} C 2 \Delta a \operatorname{lr} B:: \mathrm{erm}$ & “ \\
\hline BKE 34430 & $\operatorname{trpC2} \Delta \mathrm{racX}:: \mathrm{erm}$ & " \\
\hline BKE09670 & trpC2 $\Delta$ dat::erm & “ \\
\hline BKE31400 & trpC2 $\Delta$ alaT::erm & “ \\
\hline BKE31930 & trpC2 $\Delta$ ald::erm & “ \\
\hline BKE 26810 & trpC2 $\triangle y r p C:: e r m$ & “ \\
\hline BKE10970 & trpC2 $\Delta y i t F:: e r m$ & “ \\
\hline KS11 & trpC2 $\triangle$ dltABCD::cat & deletion of dltABCD genes \\
\hline KS21 & $\operatorname{trpC2} \Delta$ alrA::zeo $\mathrm{pLOSS} * a l r A$ & $\begin{array}{c}\mathrm{pLOSS} * \text { alrA transformed into } \\
\text { RD180 }\end{array}$ \\
\hline KS22 & $\operatorname{trpC2} \Delta$ datA::erm & $\begin{array}{l}\text { 168CA transformed with gDNA of } \\
\text { BKE30530 }\end{array}$ \\
\hline KS23 & trpC2 $\triangle$ datA::erm $\triangle$ alrA::zeo pLOSS*alrA & $\begin{array}{l}\text { KS21 transformed with gDNA of } \\
\text { BKE30530 }\end{array}$ \\
\hline KS26 & trpC2 $\Delta$ datA::erm $\triangle a m y E$ (cat $P_{\text {spac }}$ datA) & pKS1 transformed into KS22 \\
\hline KS27 & $\operatorname{trpC2} \Delta$ alrA::zeo $\triangle a m y E$ (cat $P_{\text {spac }}$ datA) & pKS1 transformed into RD180 \\
\hline KS32 & $\operatorname{trp} C 2 \Delta$ alr $A:: z e o \Delta$ alrB:::erm & $\begin{array}{l}\text { RD180 transformed with gDNA of } \\
\text { BKE17640 }\end{array}$ \\
\hline KS33 & $\operatorname{trp} C 2 \Delta a \operatorname{lr} A:: z e o \Delta r a c X:: e r m$ & $\begin{array}{l}\text { RD180 transformed with gDNA of } \\
\text { BKE34430 }\end{array}$ \\
\hline KS34 & $\operatorname{trp} C 2 \Delta a \operatorname{lr} A:: z e o \Delta y r p C:: e r m$ & $\begin{array}{l}\text { RD180 transformed with gDNA of } \\
\text { BKE } 26810\end{array}$ \\
\hline KS35 & $\operatorname{trp} C 2 \Delta$ alrA::zeo $\Delta y i t F:: e r m$ & $\begin{array}{l}\text { RD180 transformed with gDNA of } \\
\text { BKE10970 }\end{array}$ \\
\hline KS36 & trpC2 $\triangle$ datA::erm $\triangle d l t A-D:: c a t$ & $\begin{array}{l}\text { KS22 transformed with gDNA of } \\
\text { KS11 }\end{array}$ \\
\hline KS41 & $\begin{array}{l}\text { trpC2 } \Delta \text { datA::erm } \triangle a m y E\left(\text { cat } P_{\text {spac }}\right. \\
\text { ftsL(RBS)- datA ) }\end{array}$ & pKS6 transformed into KS22 \\
\hline
\end{tabular}




\begin{tabular}{|c|c|c|}
\hline KS42 & $\begin{array}{l}\text { trpC2 } \Delta \text { datA::erm } \triangle a m y E \text { (cat } P_{\text {spac }} \\
\text { ftsL(RBS)-datA) } \Delta \text { alrA::zeo }\end{array}$ & $\begin{array}{l}\text { KS41 transformed with gDNA of } \\
\text { RD180 }\end{array}$ \\
\hline KS78 & trpC2 $\Delta$ alrA::zeo $\Delta$ dat::erm & $\begin{array}{l}\text { RD180 transformed with gDNA of } \\
\text { BKE09670 }\end{array}$ \\
\hline KS79 & trpC2 $\Delta$ alrA::zeo $\Delta$ ald::erm & $\begin{array}{l}\text { RD180 transformed with gDNA of } \\
\text { BKE31390 }\end{array}$ \\
\hline MC1 & $\Delta a l a T:: e r m$ & $\begin{array}{l}\text { gDNA of BKE31400 transformed } \\
\text { into } 168+.\end{array}$ \\
\hline MC2 & $\Delta a l a T$ & $\begin{array}{l}\text { Erythromycin marker was removed } \\
\text { using pDR244. }\end{array}$ \\
\hline MC3 & $\Delta$ dat::erm & $\begin{array}{l}\text { gDNA of BKE09670 transformed } \\
\text { into } 168+.\end{array}$ \\
\hline MC4 & $\Delta$ alaT $\Delta$ dat::erm & $\begin{array}{l}\text { gDNA of BKE } 09670 \text { transformed } \\
\text { into MC2. }\end{array}$ \\
\hline MC5 & $\Delta$ ald::erm & $\begin{array}{l}\text { gDNA of BKE } 09670 \text { transformed } \\
\text { into } 168+.\end{array}$ \\
\hline MC6 & $\Delta$ alaT $\Delta$ ald::erm & $\begin{array}{l}\text { gDNA of BKE } 09670 \text { transformed } \\
\text { into MC2 }\end{array}$ \\
\hline MC7 & $\Delta$ datA::erm & $\begin{array}{l}\text { gDNA of BKE } 09670 \text { transformed } \\
\text { into } 168+\text {. }\end{array}$ \\
\hline MC8 & $\Delta$ alaT $\Delta$ datA::erm & $\begin{array}{l}\text { gDNA of BKE } 09670 \text { transformed } \\
\text { into MC2. }\end{array}$ \\
\hline $\begin{array}{l}\text { E. coli } \\
\text { strains }\end{array}$ & Genotype/Relevant features & $\begin{array}{c}\text { Construction or Source or } \\
\text { Reference }\end{array}$ \\
\hline DH5 $\alpha$ & $\begin{array}{l}\text { fhuA2 } \triangle(\arg F-l a c Z) U 169 \text { phoA glnV44 } \Phi 80 \\
\Delta(\text { lacZ)M15 gyrA96 recA1 relA1 endA1 thi-1 } \\
\text { hsdR17 }\end{array}$ & New England BioLabs ${ }^{\circledR}$ \\
\hline
\end{tabular}

Table S1. Plasmid constructs used in this study.

\begin{tabular}{|c|c|c|c|}
\hline Name plasmid & Relevant features & Construction & Source/Reference \\
\hline pPY18 & $\begin{array}{c}\text { bla amyE3' cat } P_{\text {spac }} \text { lacI } \\
\text { amyE5' }\end{array}$ & ------ & (53) \\
\hline pSG1 & bla cat & ------- & (55) \\
\hline pLOSS*alrA & $\begin{array}{c}\text { bla spc } P_{\text {spac }} \text { alrA } A^{W 23} \text { - } \\
\text { PdivlA- lacZ lacI rep } \\
\text { pLS20* }\end{array}$ & $\begin{array}{l}\text { Inserstion of alrA } \\
\text { gene into pLOSS* } \\
\text { vector }\end{array}$ & This work \\
\hline
\end{tabular}




\begin{tabular}{|c|c|c|c|}
\hline pKS1 & $\begin{array}{c}\text { bla amyE3' cat } P_{\text {spac }} \text { datA } \\
\text { lacI amyE5' }\end{array}$ & $\begin{array}{c}\text { Insertion of datA } \\
\text { gene and its } \\
\text { upstream } 30 \text { bases } \\
\text { into XbaI cut } \\
\text { pPY18 }\end{array}$ & This work \\
\hline pKS6 & $\begin{array}{l}\text { bla amyE3' cat } P_{\text {spac }} \text { datA- } \\
\text { ftsL(RBS) lacI lacZ amyE5' }\end{array}$ & $\begin{array}{l}\text { Insertion of datA } \\
\text { gene and RBS of } \\
f t s L \text { gene into } \\
\text { XbaI and BglIIcut } \\
\text { pPY18 }\end{array}$ & This work \\
\hline
\end{tabular}

\section{Supplemental figures, methods and materials}

\section{Figure S1}

Standard curve for D-alanine quantification in LB media. A) The RP-HPLC analysis of 2000 MWCO filtered LB medium with and without D-alanine $(\mathrm{mM})$. The red arrows indicate the D-alanine peaks, which were raised around $34 \mathrm{~min}$. B) A standard curve was generated from the data of figure (A) for quantitative determination of $\mathrm{D}$-alanine in the growth medium.

\section{Figure S2}

RP-HPLC analysis for the detection of D-alanine in culture supernatants.

Panel A - Growth curves for cultures samples for HPLC analysis (panel B) the wild-type strain $(\bullet)$ 168CA), $\Delta \operatorname{dat} A(\mathrm{O} ; \mathrm{KS} 22), \Delta d \mathrm{dtA}-\mathrm{D}(\boldsymbol{\nabla} ; \mathrm{KS} 11)$ and $\Delta d \mathrm{dtA}-\mathrm{D} \operatorname{dat} A(\triangle ; \mathrm{KS} 36)$ strains in LB medium at $37^{\circ} \mathrm{C}$. The black arrows represent the time points, when the samples were taken for RP-HPLC analysis.

Panel B - RP-HPLC analysis of 2000 MWCO filtered culture supernatants of 168CA and $\triangle$ datA (KS22) strains at different time points $(90,180,390 \mathrm{~min}$ and $\mathrm{O} / \mathrm{N})$. 
Panal C - RP-HPLC analysis of $\Delta d l t A-D$ (KS11) and $\Delta d l t A-D \Delta d a t A$ (KS36) strains at 180 min of incubation.

The red arrows indicate D-alanine peaks, which appeared at around $34 \mathrm{~min}$.

\section{Figure S3}

Complementation and Transcription analysis of $\operatorname{datA}$ gene.

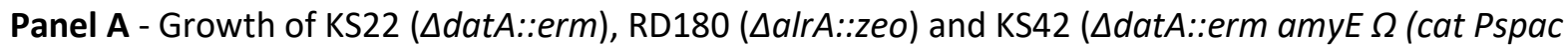
datA-ftsL(RBS)) $\Delta a l r A:: z e o$ ) strains on nutrient agar (NA) plates with and without $1.0 \mathrm{mM}$ IPTG and 0.5 $\mathrm{mM}$ D-alanine. The plates were incubated at $37^{\circ} \mathrm{C}$ overnight.

Panel B provides a diagrammatic representation of the chromosomal region up and downstream of the datA gene below which are shown numbered DNA fragments which were amplified by PCR. The numbers indicate which specific pair of oligonucleotides (sequences of which can be found in Table S2) were used to generate the DNA fragment. The images of agarose gel show products of PCR reactions (32 cycles), using gDNA (panel C) and cDNA (panel D) as templates. The lane numbers correspond to the numbered bars in panel $\mathrm{A}$.

\section{Figure S4}

Genes involved in the use of alanine as sole carbon/nitrogen source. For testing alanine used as sole carbon source, $\Delta$ alaT (MC2) $\Delta$ dat (MC3) and $\Delta$ ald (MC5) were grown on M plates with $25 \mathrm{mM} \mathrm{D-/L-}$ alanine. For testing alanine used as nitrogen source, these three strains were grown on $\mathrm{GM}-\mathrm{NH}_{3}$ plates supplemented with $25 \mathrm{mM} \mathrm{D}-/ \mathrm{L}$-alanine. The plates were incubated at $37^{\circ} \mathrm{C}$ for 48 hours.

\section{Real-time PCR assay}

The wild type strain (168CA) was grown in LB media to mid-exponential growth $\left(\mathrm{OD}_{600} \sim 1.0\right)$ at $37{ }^{\circ} \mathrm{C}$. Total RNA was extracted from $1.0 \mathrm{ml}$ the culture, using total RNA purification plus Kit (NORGEN Biotek 
Corp.) according to the manufacturer’s protocol. The high capacity cDNA reverse transcription kit (Applied Biosystems $^{\mathrm{TM}}$ ) was used for making cDNA according to manufacturer's protocol. The total RNA extract was used as template and non-specific random hexamers as primer in cDNA production. Real-time PCR was then performed on normal PCR machine, using DNA as template, gene specific primers and Q5 ${ }^{\mathrm{TM}}$ High-Fidelity DNA polymerase (New England BioLabs ${ }^{\circledR}$ ) according to the manufacturer’s instructions. The Real-time PCR products were run on agarose gel (Fig S2).

\section{Table S2. Oligonucleotides}

\begin{tabular}{|c|c|c|c|}
\hline Name & Sequence & $\begin{array}{c}\text { Restriction } \\
\text { site }\end{array}$ & Purpose \\
\hline alrAW23-1 & $\begin{array}{l}\text { 5’-ATCTCTAGACGTTAGGCATCGTTTC } \\
\text { CCTTTGTCC-`3 }\end{array}$ & XbaI & \multirow{2}{*}{$\begin{array}{l}\text { Construction of } \\
\mathrm{p} L O S S^{*} \text { alrA } A^{W 23}\end{array}$} \\
\hline alrAW23-2 & $\begin{array}{l}\text { 5’- ACTGGTACCCTATTTGCTTGTATTT } \\
\text { TCCTGCAATAAAGGATTTC }\end{array}$ & BamHI & \\
\hline oKS01 & 5'-AAGGAACAGATGATGCGCACG-'3 & --------- & \multirow{5}{*}{$\begin{array}{l}\text { For deletion of } \\
\text { dltABCD } \\
\text { genes }\end{array}$} \\
\hline oKS02 & $\begin{array}{l}\text { 5’AGTTCTAGATCCGCATGTGTTTGAA } \\
\text { TAGC-'3 }\end{array}$ & Xbal & \\
\hline oKS03 & $\begin{array}{l}\text { 5'ATATCTAGAAGATCTAGCGAAGGG } \\
\text { CTTCCAGGTTGC-'3 }\end{array}$ & $\begin{array}{c}\text { Xbal and } \\
\text { BglII }\end{array}$ & \\
\hline oKS04 & 5'-TGCGATTTCTCCTGTTTCACCG-'3 & --------- & \\
\hline oKS12 & 5'-TAGTATTCGGCACAGATCGGC-'3 & --------- & \\
\hline oKS21 & $\begin{array}{l}\text { 5'ATCTCTAGAAATGATATTTTTTTCTA } \\
\text { GGGGAGAAGAAGC-'3 }\end{array}$ & $X b a \mathrm{I}$ & \multirow{2}{*}{$\begin{array}{c}\text { For pKS1 } \\
\text { construction }\end{array}$} \\
\hline oKS22 & $\begin{array}{l}\text { 5’TGATCTAGATCAGCTGATATTTCGT } \\
\text { TCGCTGGC-'3 }\end{array}$ & $X b a \mathrm{I}$ & \\
\hline oKS25 & $\begin{array}{l}\text { 5’ATCTCTAGAAAAATTAAAAGGAGG } \\
\text { TCATCAGCCTATGCAAAAACAAAAAC } \\
\text { AAGAGCTGCACCGC-'3 }\end{array}$ & $X b a \mathrm{I}$ & \multirow{2}{*}{$\begin{array}{c}\text { For pKS6 } \\
\text { construction }\end{array}$} \\
\hline oKS26 & $\begin{array}{l}\text { 5’TGAAGATCTTCAGCTGATATTTCGT } \\
\text { TCGCTGGC-'3 }\end{array}$ & BglII & \\
\hline oKS38 & 5'-AACGGCTCGGTGACATGTAT-'3 & --------- & \\
\hline oKS39 & 5'-TGTTCAATGGCTTTGATCGT-'3 & --------- & \\
\hline
\end{tabular}




\begin{tabular}{|c|c|c|c|}
\hline oKS40 & 5'-GGACTTGGACCAGCAAACAG-'3 & --------- & \multirow{30}{*}{$\begin{array}{c}\text { oKS38 } \\
\text { to } \\
\text { oKS69 } \\
\text { used for } \\
\text { transcription } \\
\text { analysis of datA } \\
\text { gene }\end{array}$} \\
\hline oKS41 & 5’-CGGAAAAACCTCCСTTTTCT-’3 & --------- & \\
\hline oKS42 & 5'-AGCGGTGCTTTCTGTGAAAT-’3 & --------- & \\
\hline oKS43 & 5'-GTGCGGCTATTATTGCGATT-’3 & -------- & \\
\hline oKS44 & 5'-TGTCCAGCTCTTGATGAACG-'3 & -------- & \\
\hline oKS45 & 5'-CAGAGAGTGGGAAGGGCTTT-'3 & -------- & \\
\hline oKS46 & 5'-GCATCACAAGAAGCGGAACT-’3 & --------- & \\
\hline oKS47 & 5'-TCGCTCCGTCACTTAAGGTT-'3 & --------- & \\
\hline oKS48 & 5'-ACTTGCCCCGTGAATGATTA-'3 & -------- & \\
\hline oKS49 & 5'-TTCTCCGATCACGACATCAC-'3 & --------- & \\
\hline oKS50 & 5'-CGGAGAACAGTCCAGCATTT-’3 & -------- & \\
\hline oKS51 & 5'-ATCCCGATTAAGGCGTTCTT-'3 & -------- & \\
\hline oKS52 & 5'-CGCCCTGCAAAAGTCATC-'3 & -------- & \\
\hline oKS53 & 5'-TTGCTGGCTGTGTACTATGGA-'3 & --------- & \\
\hline oKS54 & 5'-TTTCTTTTCTTCCGGCTTCA-'3 & -------- & \\
\hline oKS55 & 5'-ATCCCGGGTAAAGCTGTTCT-'3 & -------- & \\
\hline oKS56 & 5'-GCGGTGCAGCTCTTGTTT-'3 & -------- & \\
\hline oKS57 & 5'-AGCTGAGCCATCAGCCTAAA-'3 & -------- & \\
\hline oKS58 & 5'-CCGTTCTTCGTTTTCAGCTC-'3 & -------- & \\
\hline oKS59 & 5'-CGCGATGTGACGAAGACTTA-'3 & -------- & \\
\hline oKS60 & 5'-GCTTGTTAAAAACCCCGACA-'3 & --------- & \\
\hline oKS61 & 5'-GGAGCAGCTGCGTAAAGAAG-'3 & --------- & \\
\hline oKS62 & 5'-GGGCAAGTGAAATTGGAAGA-'3 & -------- & \\
\hline oKS63 & 5'-ATCGGATACACACGTGCAAA-'3 & -------- & \\
\hline oKS64 & 5'-GCCCCTCCGTAACAGATTC-'3 & -------- & \\
\hline oKS65 & 5'-TGAAAGGCACAAAGACCAAA-'3 & -------- & \\
\hline oKS66 & 5'-TCGTAAAAGGGTTTGCAATG-'3 & -------- & \\
\hline oKS67 & 5'-CAGCGGTCAAATCAACTGAG-'3 & -------- & \\
\hline oKS68 & 5'AGCGGCTGTACACGAAAGTT-'3 & -------- & \\
\hline oKS69 & 5'-AACGGCTGCTCAACTGTTTT-'3 & -------- & \\
\hline
\end{tabular}

- $\quad$ The restriction sites are shown as bold, underlined letters. 
Table S3 Pump and run parameters of RP-HPLC analysis

\begin{tabular}{|c|c|c|c|c|c|c|c|c|}
\hline Step & Time (min) & $\begin{array}{c}\text { Flow } \\
(\mathbf{m l} / \mathbf{m i n})\end{array}$ & A & B & C & D & Curve & $\begin{array}{c}\text { Sample } \\
\text { volume( } \boldsymbol{\mu l})\end{array}$ \\
\hline 0 & 0.5 & 1.00 & 90.0 & 10.0 & 0.0 & 0.0 & 0.0 & \\
\hline 1 & 1.5 & 1.00 & 90.0 & 10.0 & 0.0 & 0.0 & 0.0 & \\
\hline 2 & 40.0 & 1.00 & 75.0 & 25.0 & 0.0 & 0.0 & 1.0 & \multirow{2}{*}{150} \\
\hline 3 & 25.0 & 1.00 & 60.0 & 40.0 & 0.0 & 0.0 & 1.0 & \\
\hline 4 & 0.5 & 1.00 & 90.0 & 10.0 & 0.0 & 0.0 & 0.0 & \\
\hline 5 & 20.0 & 1.00 & 90.0 & 10.0 & 0.0 & 0.0 & 0.0 & \\
\cline { 1 - 2 } $\begin{array}{c}\text { Total } \\
\text { Run Time }\end{array}$ & 87.00 & & & & & & &
\end{tabular}


bioRxiv preprint doi: https://doi.org/10.1101/562850; this version posted April 18, 2019. The copyright holder for this preprint (which was not certified by peer review) is the author/funder. All rights reserved. No reuse allowed without permission.

\begin{tabular}{|c|c|}
\hline Strains & Geneotype \\
\hline MGNA-C343 & trpC2 $\Delta y f k T:: p M U T I N 4$ (erm) \\
\hline MGNA-B989 & $\operatorname{trpC2} \Delta y c g H:: p M U T I N 4$ (erm) \\
\hline MGNA-B757 & trpC2 $\Delta y w a A:: p M U T I N 4$ (erm) \\
\hline MGNA-C001 & trpC2 $\Delta y c k A:: p M U T I N 4$ (erm) \\
\hline MGNA-C394 & trpC2 $\Delta y q i N:: p M U T I N 4$ (erm) \\
\hline MGNA-C383 & trpC2 $\Delta y q i Z:: p M U T I N 4$ (erm) \\
\hline MGNA-C480 & trpC2 $\Delta y q g T:$ :pMUTIN4 (erm) \\
\hline MGNA-A172 & trpC2 $\Delta y t m L:: p M U T I N 4$ (erm) \\
\hline MGNA-B963 & trpC2 $\Delta y b e C:: p M U T I N 4$ (erm) \\
\hline MGNA-B783 & trpC2 $\Delta$ hutM::pMUTIN4 (erm) \\
\hline MGNA-B940 & $\operatorname{trpC2} \Delta y b g F:: p M U T I N 4$ (erm) \\
\hline MGNA-C381 & trpC2 $\Delta y q i X:$ :pMUTIN4 (erm) \\
\hline MGNA-B939 & trpC2 $\Delta y b g E:: p M U T I N 4(\mathrm{erm})$ \\
\hline MGNA-B914 & trpC2 $\Delta$ yabM::pMUTIN4 (erm) \\
\hline MGNA-C084 & trpC2 $\Delta y d g F:: p M U T I N 4(\mathrm{erm})$ \\
\hline MGNA-C022 & $\operatorname{trpC2} \Delta y c s G:: p M U T I N 4$ (erm) \\
\hline MGNA-A551 & trpC2 $\Delta y u r H:: p M U T I N 4$ (erm) \\
\hline MGNA-A736 & trpC2 $\Delta y k b A:: p M U T I N 4$ (erm) \\
\hline MGNA-A031 & trpC2 $\Delta y j o B:: p M U T I N 4$ (erm) \\
\hline MGNA-A468 & $\operatorname{trpC2} \Delta y v b W:: \mathrm{pMUTIN} 4$ (erm) \\
\hline MGNA-A012 & trpC2 $\Delta y \operatorname{tg} P:: p M U T I N 4$ (erm) \\
\hline MGNA-A909 & trpC2 $\Delta$ уесA::pMUTIN4 (erm) \\
\hline MGNA-A362 & trpC2 $\Delta y j k B:: p M U T I N 4$ (erm) \\
\hline MGNA-A311 & $\operatorname{trpC2} \Delta y o b N:: p M U T I N 4$ (erm) \\
\hline MGNA-A173 & trpC2 $\Delta y t m M:: p M U T I N 4$ (erm) \\
\hline MGNA-A230 & trpC2 $\Delta a a p A:: p M U T I N 4$ (erm) \\
\hline MGNA-B479 & trpC2 $\Delta y h d G:: p M U T I N 4$ (erm) \\
\hline MGNA-C382 & trpC2 $\Delta y q i Y:: p M U T I N 4$ (erm) \\
\hline MGNA-A171 & trpC2 $\Delta y t m K:: p M U T I N 4$ (erm) \\
\hline MGNA-C231 & trpC2 $\Delta y f n A:: p M U T I N 4$ (erm) \\
\hline MGNA-B782 & trpC2 $\triangle$ ухеM::pMUTIN4 (erm) \\
\hline MGNA-A126 & trpC2 $\Delta y \operatorname{tnA::pMUTIN4~(erm)~}$ \\
\hline MGNA-A595 & trpC2 $\Delta y u s A:: p M U T I N 4$ (erm) \\
\hline MGNA-A596 & trpC2 $\Delta y u s C:: p M U T I N 4$ (erm) \\
\hline MGNA-A686 & trpC2 $\Delta y h a G:: p M U T I N 4$ (erm) \\
\hline MGNA-C257 & trpC2 $\Delta y f l A:: p M U T I N 4$ (erm) \\
\hline MGNA-A659 & trpC2 $\Delta y h c G:: p M U T I N 4$ (erm) \\
\hline MGNA-A653 & trpC2 $\Delta y h c H:: p M U T I N 4$ (erm) \\
\hline MGNA-A655 & trpC2 $\Delta y h c J:: p M U T I N 4$ (erm) \\
\hline MGNA-A834 & trpC2 $\Delta$ yoaC::pMUTIN4 (erm) \\
\hline MGNA-A835 & trpC2 $\Delta$ yoaD::pMUTIN4 (erm) \\
\hline
\end{tabular}




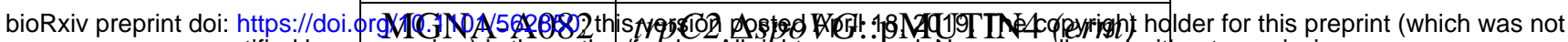
certified by peer review) is the author/funder. All rights reserved. No reuse allowed without permission. 
bioRxiv preprint doi: https://doi.org/10.1101/562850; this version posted April 18, 2019. The copyright holder for this preprint (which was not certified by peer review) is the author/funder. All rights reserved. No reuse allowed without permission. 
Fig. 1
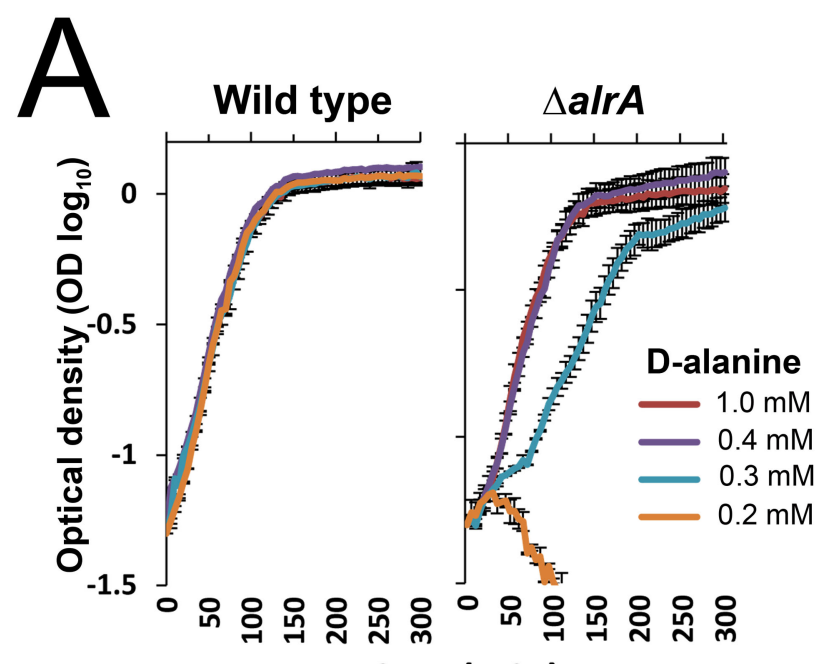

B

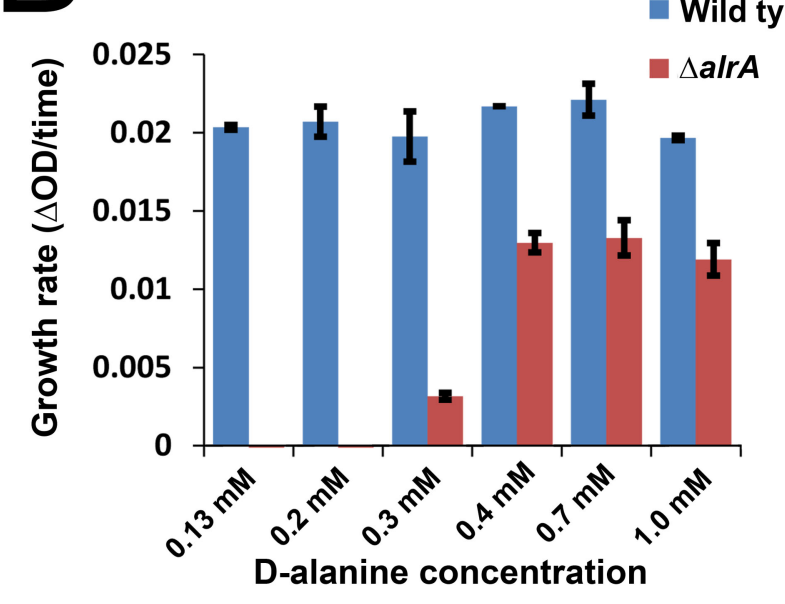

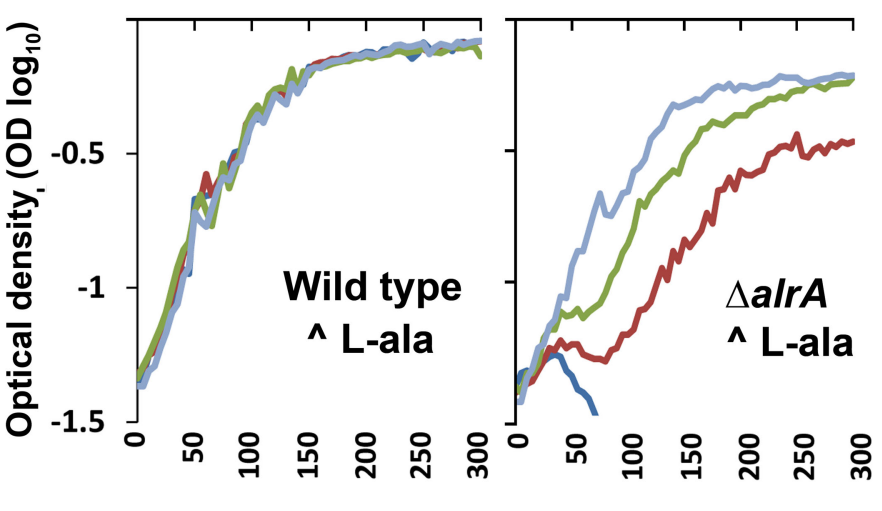

Time (min)

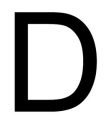

LB medium + $0.45 \mathrm{mM}$ D-alanine

no addition

1.25 mM L-alanine

$2.5 \mathrm{mM}$ L-alanine

$5.0 \mathrm{mM}$ L-alanine

\section{Time (min)}

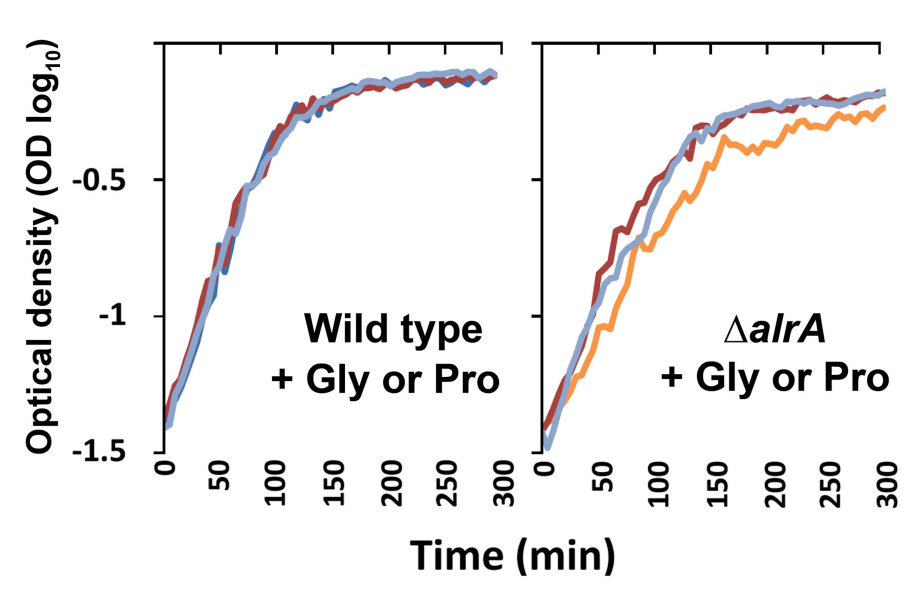

LB medium + 0.45 mM D-alanine

no addition

- $5 \mathrm{mM}$ glycine

- $5 \mathrm{mM}$ L-proline 
Fig 2

A wild type

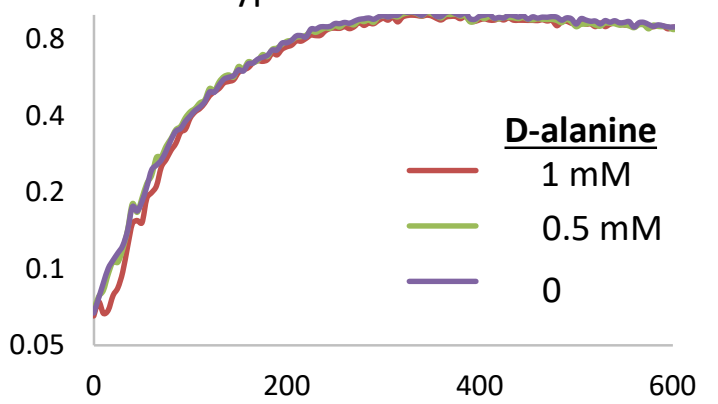

B

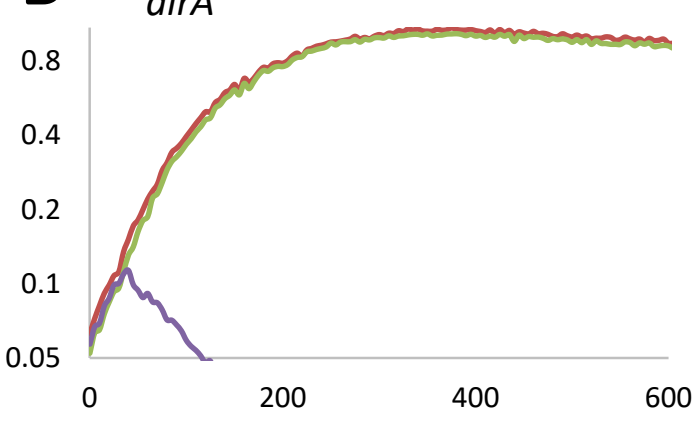

C

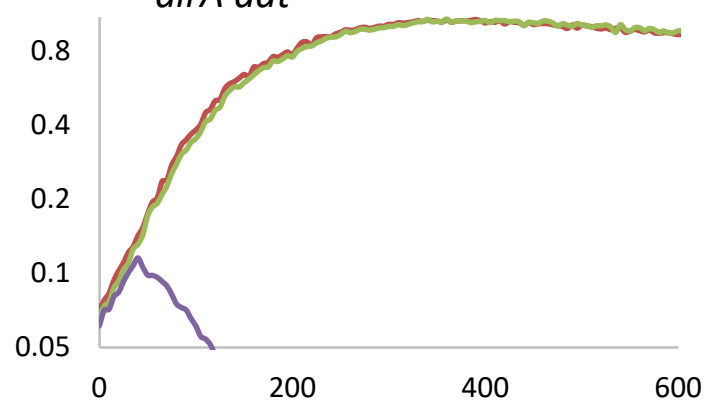

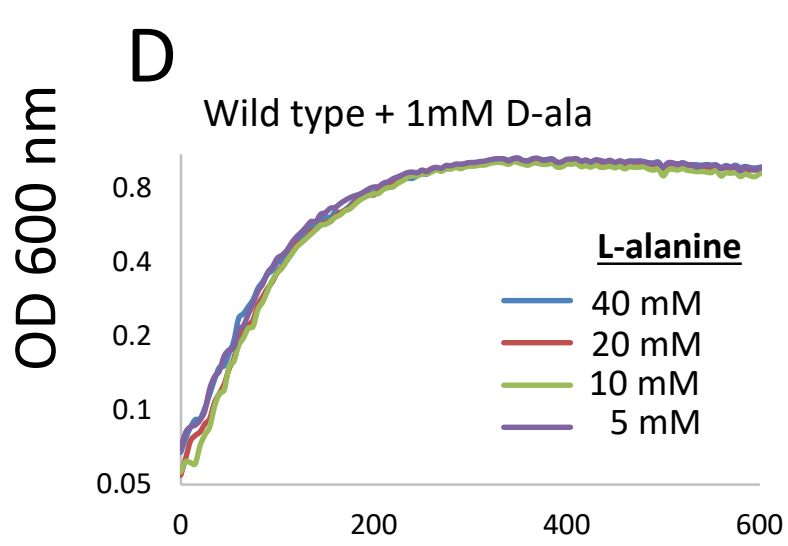

E

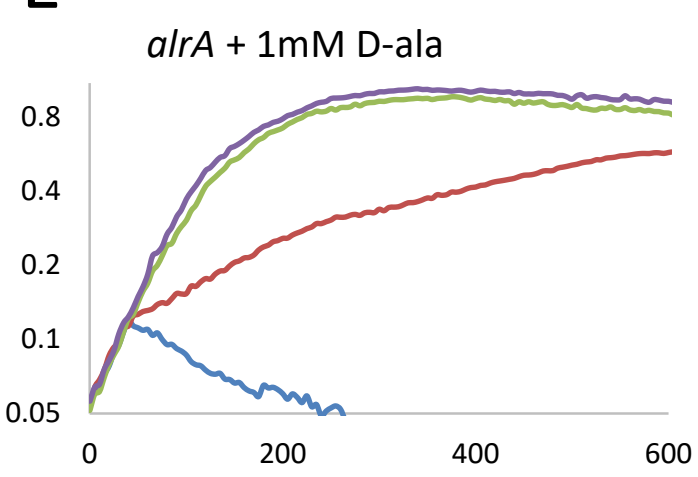

F

alrA dat + 1mM Dala

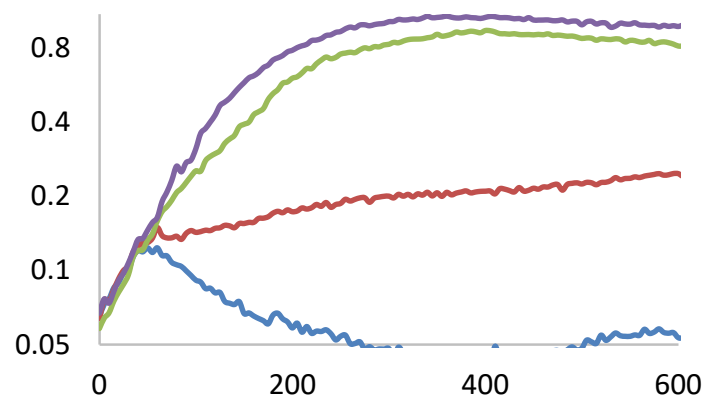

G Wild type

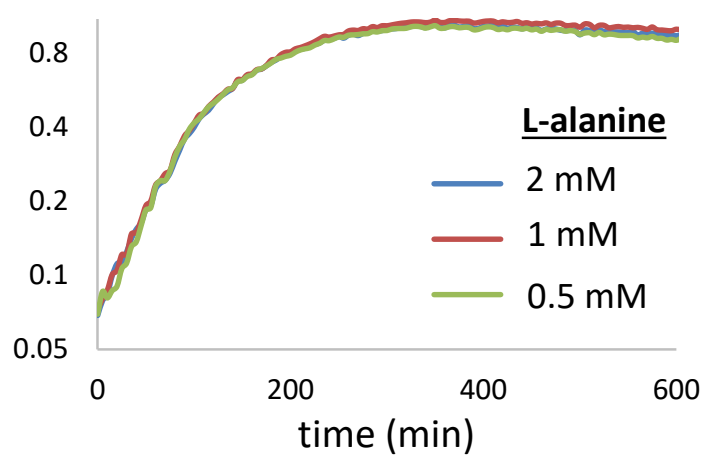

\section{$\mathrm{H}$ alra}

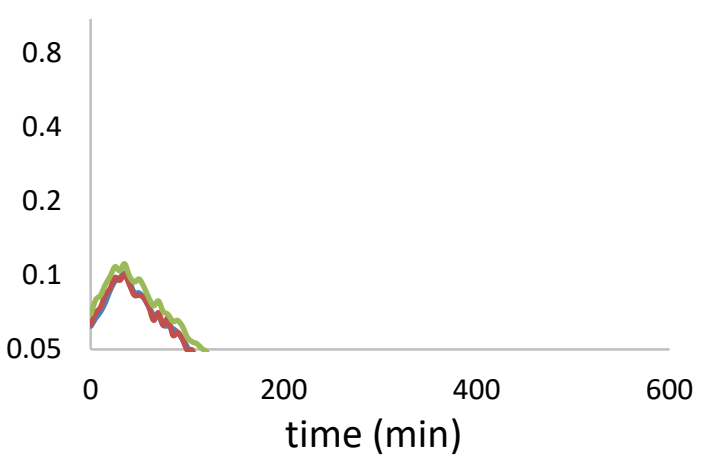

alrA dat

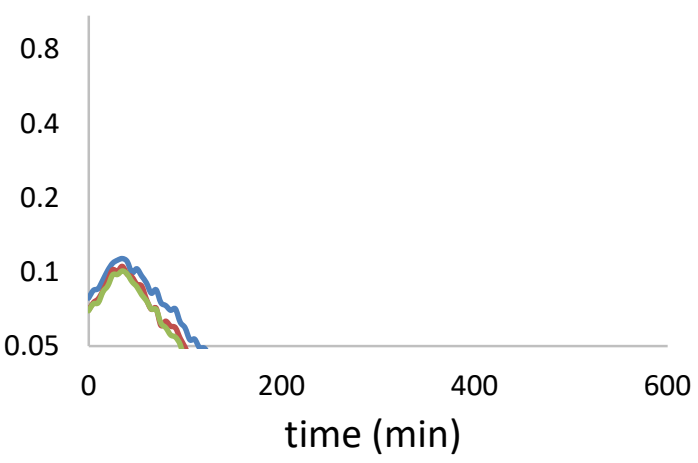


Fig. 3

A
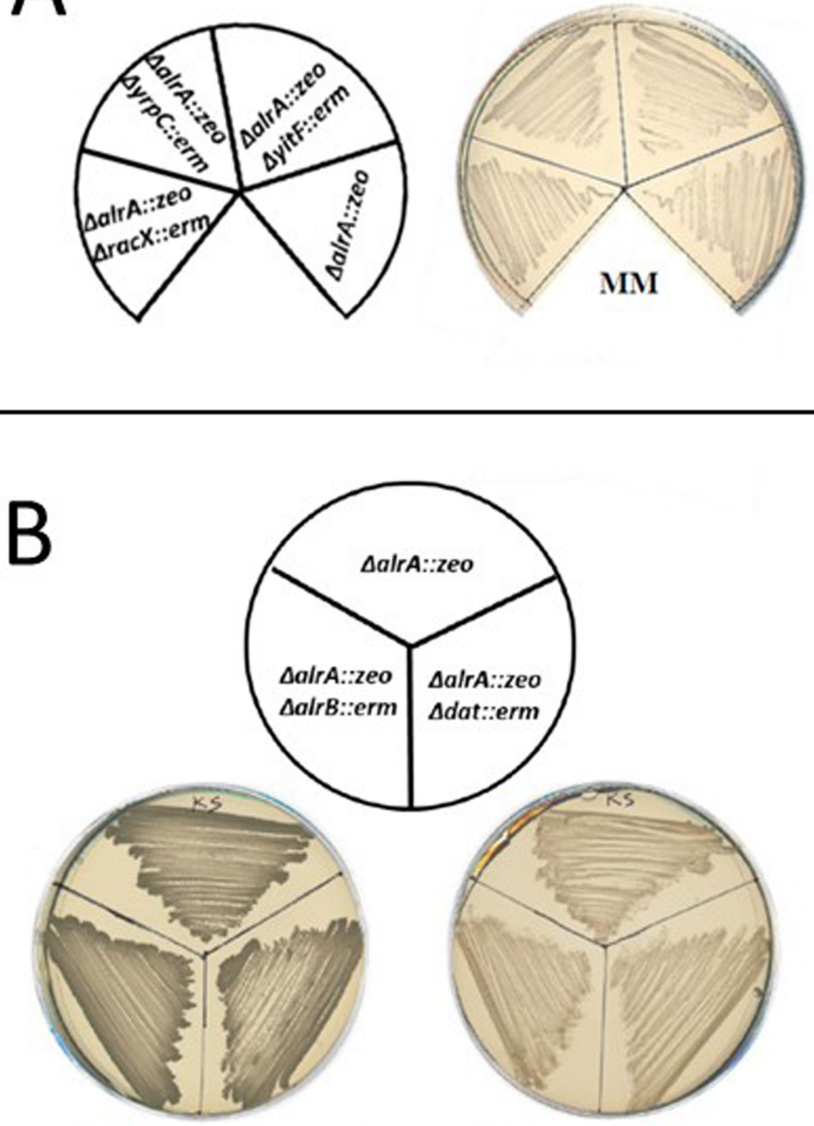

$\mathrm{NA}+0.5 \mathrm{mM}$ D-alanine
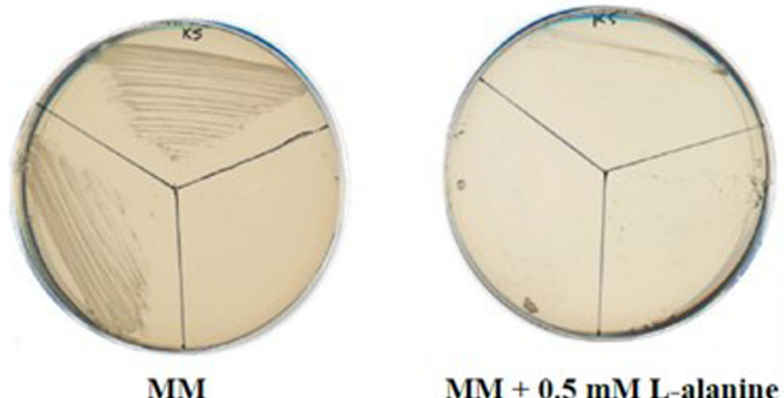

$\mathrm{MM}+0.5 \mathrm{mM}$ L-alanine 
Fig 4
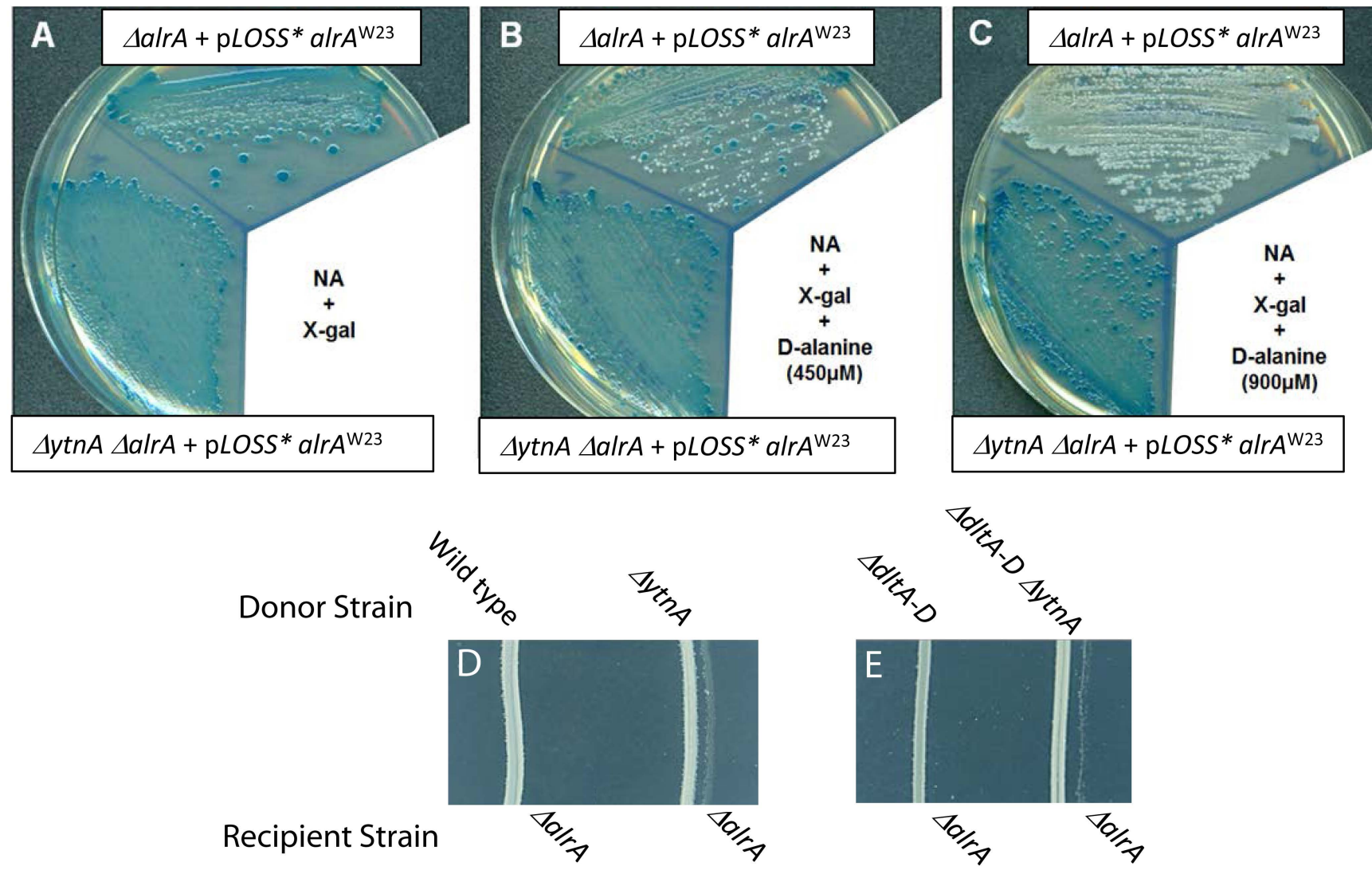
Fig. 5

\section{Detection of extracellular alanine during growth}

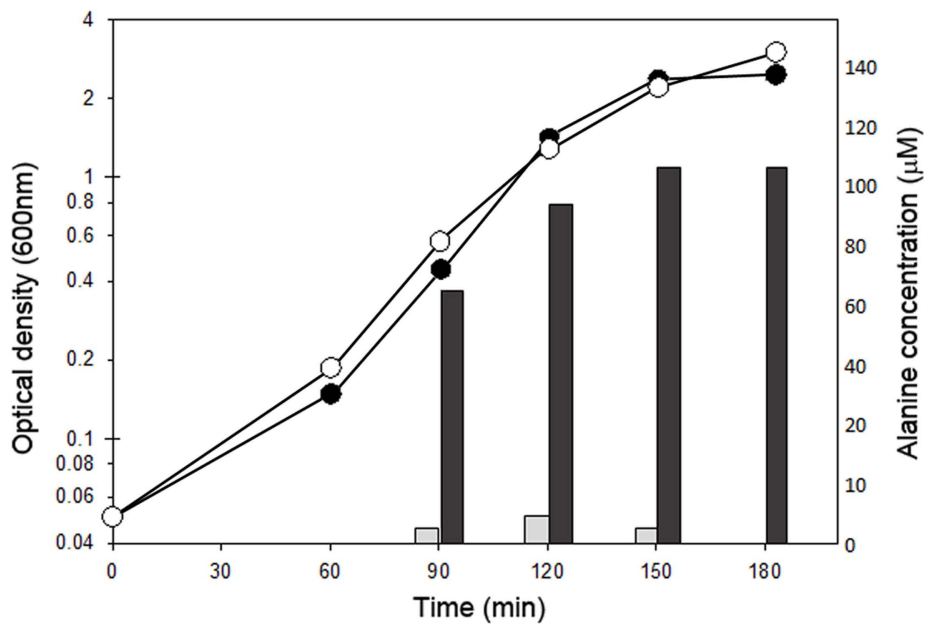

Culture optical density Free Alanine concentration $(\mu \mathrm{M})$

- Wild-type

- - $\triangle$ datA

Wild-type media

$\triangle$ datA media

Depletion of extracellular D- or L-alanine during growth

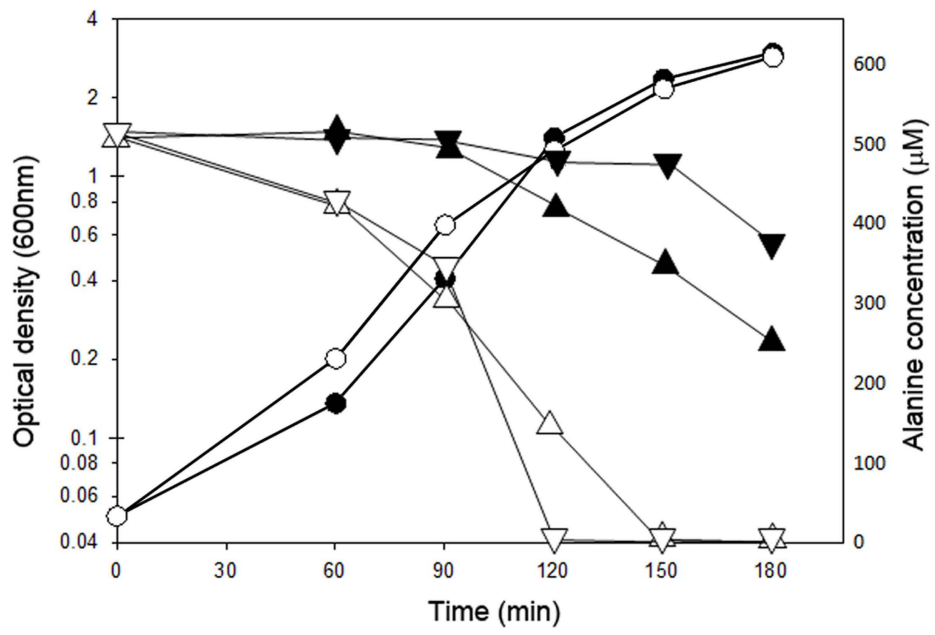

Culture optical density Free Alanine concentration $(\mu \mathrm{M})$

- -Wild-type

D-alanine in Wild-type culture $\triangle ; \Delta$ dat $A$ culture

$-\mathrm{-} \Delta$ datA

L-alanine in Wild-type culture $\nabla ; \Delta$ dat $A$ culture 
Fig 6.

A

B

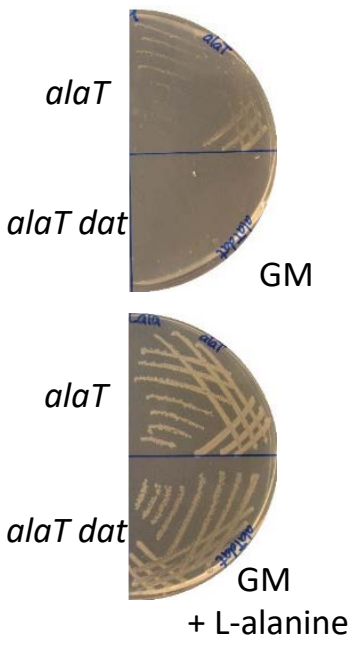

C

Concentration of added alanine

$-1 \mathrm{mM}$
$-0.8 \mathrm{~mm}$

$-0.6 \mathrm{mM}$
$-0.5 \mathrm{mM}$

$-0.5 \mathrm{mM}$
$-0.4 \mathrm{mM}$

- No addition

(iii)

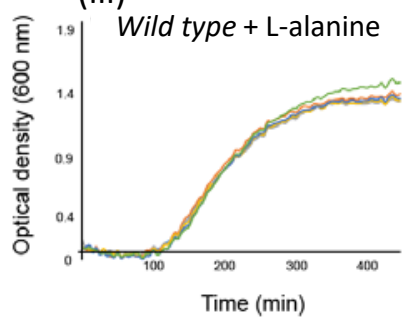

(i)

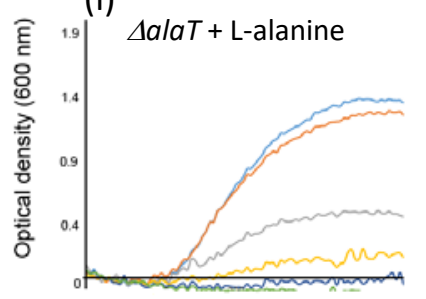

(iv)

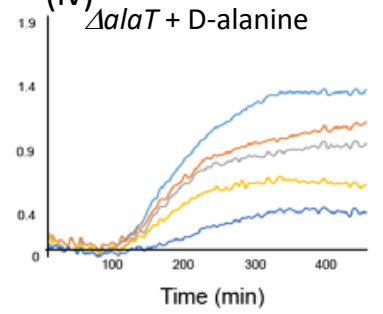

(ii)
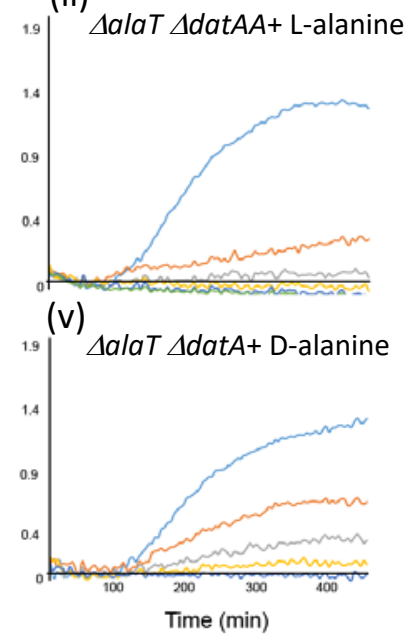


\section{Fig. S1. Standard curve for D-alanine quantification in}

\section{LB media}

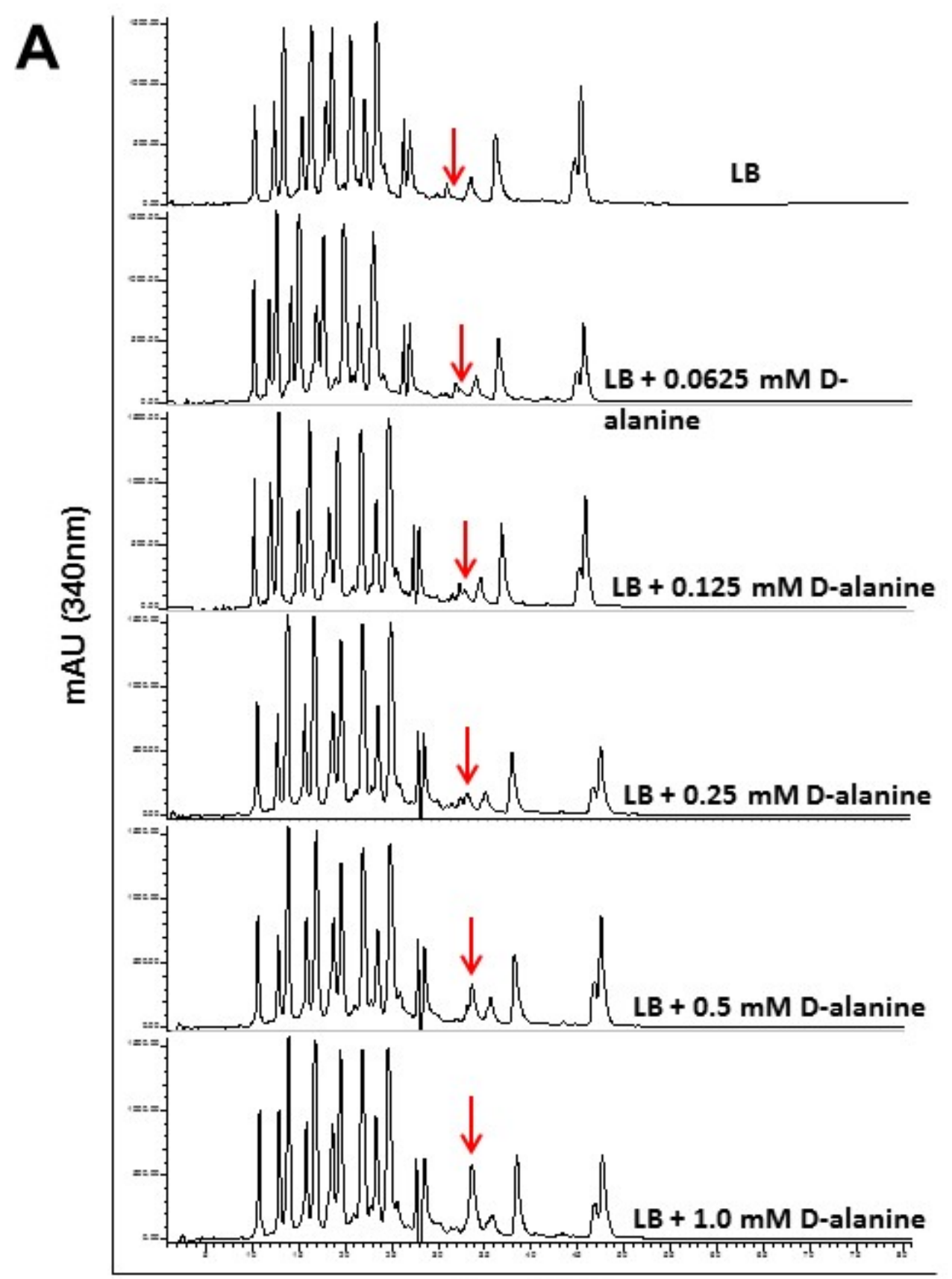

Time (min)

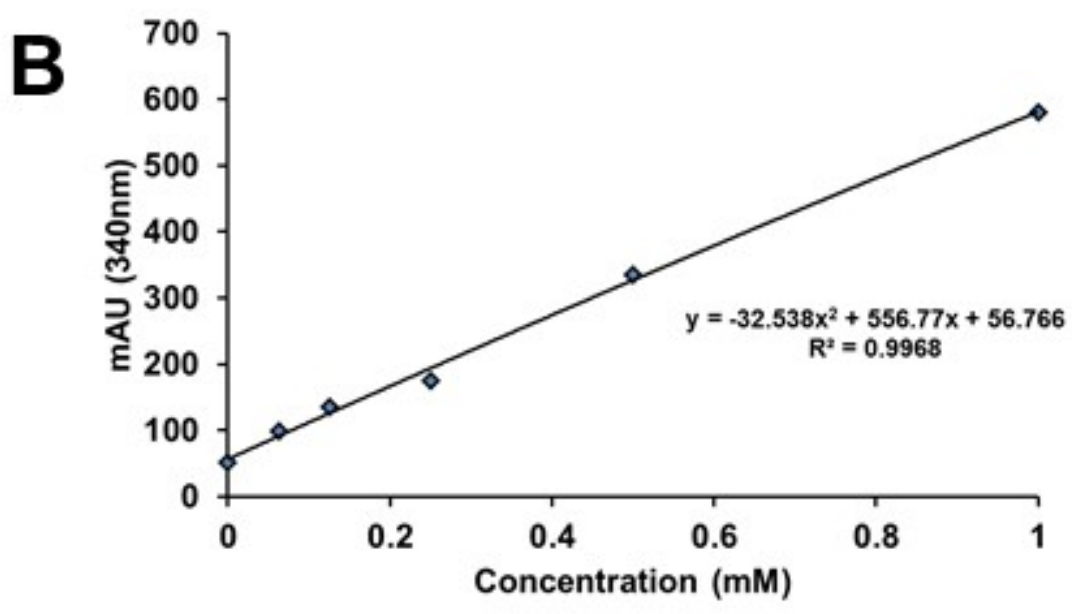


Fig S2

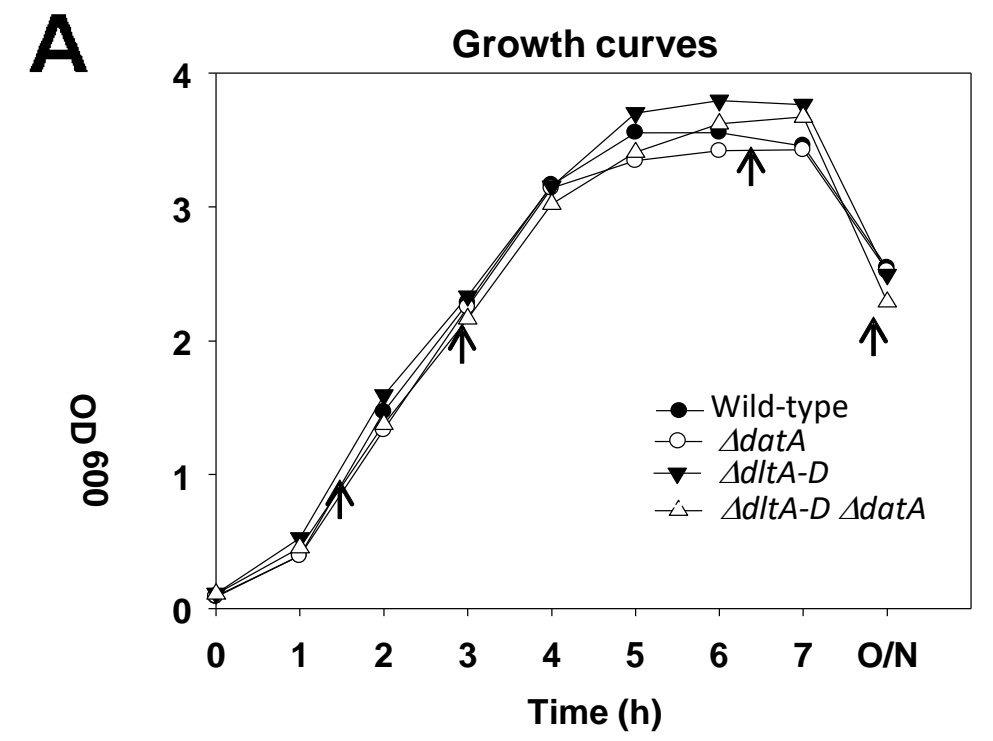

B

Wild-type

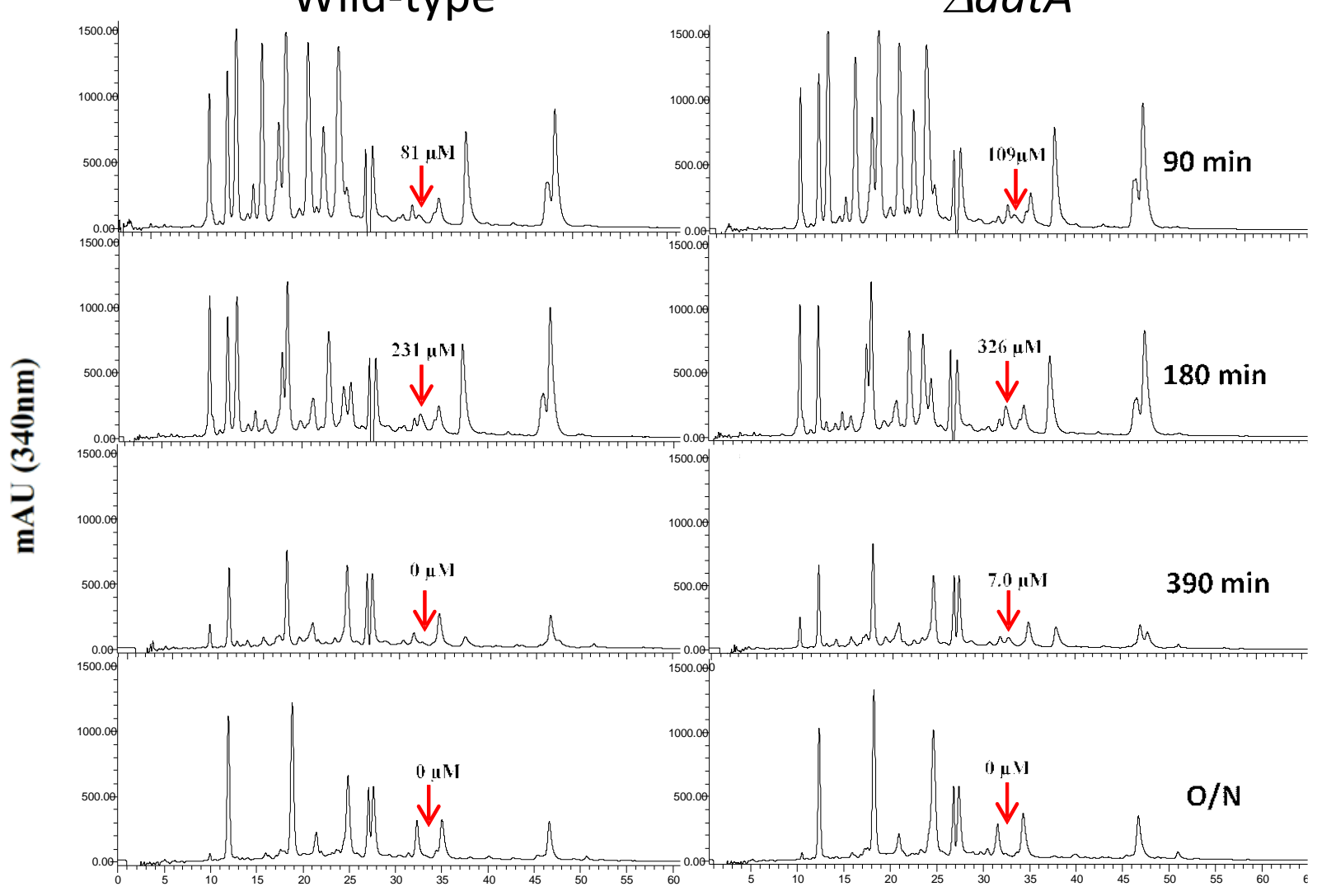

Time (minute)

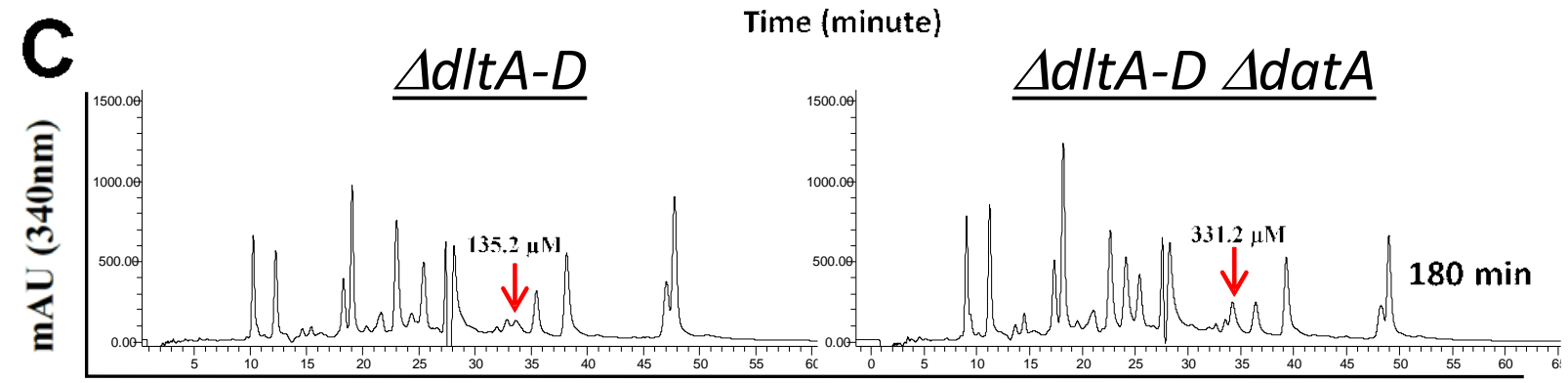

Time (minute) 
Fig. S3

A

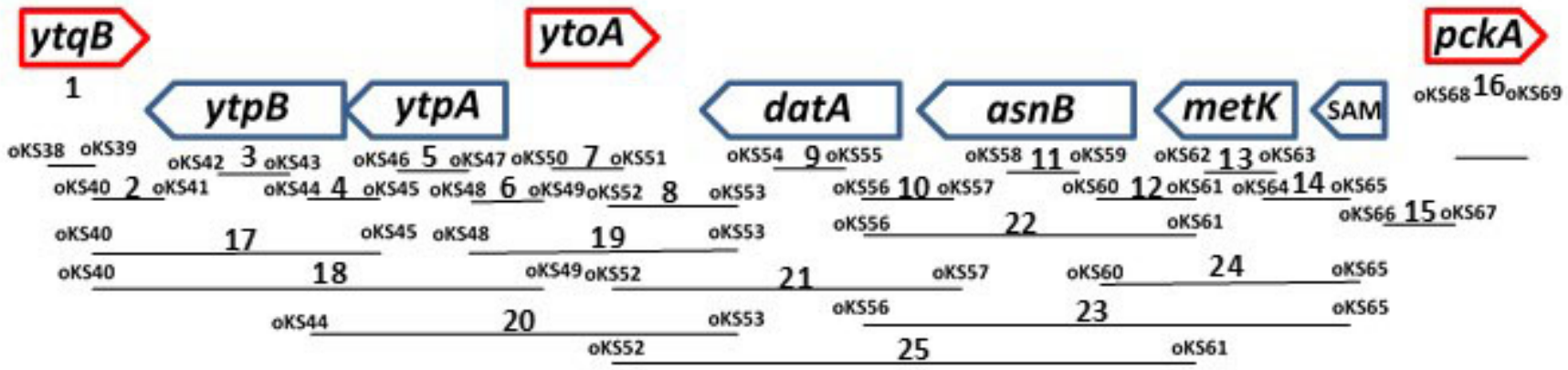

B

$\underline{K b}$

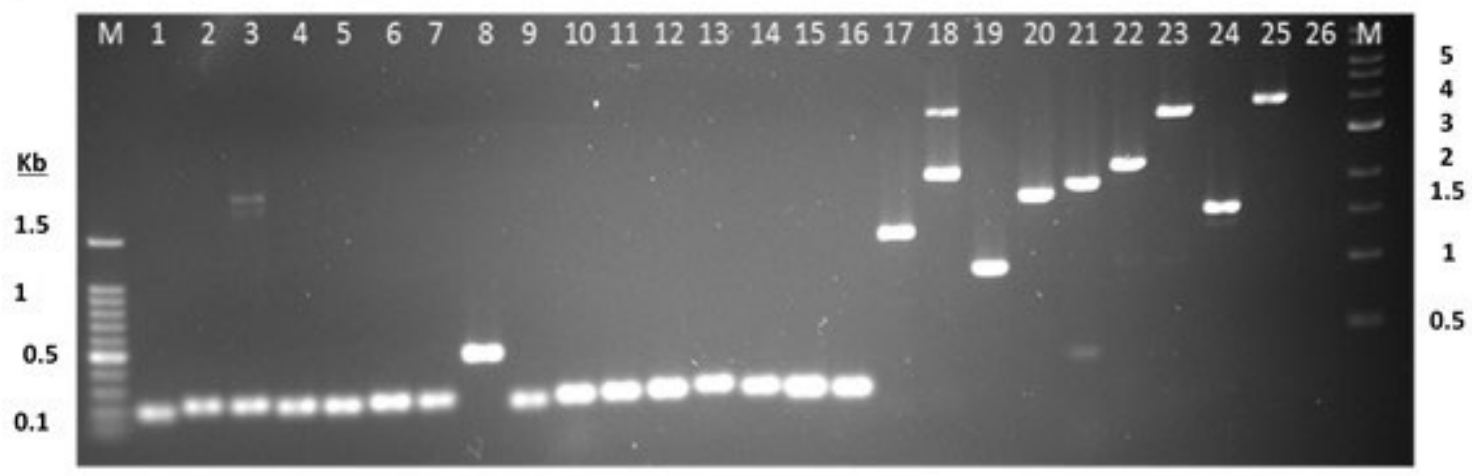

C

$\mathrm{Kb}$

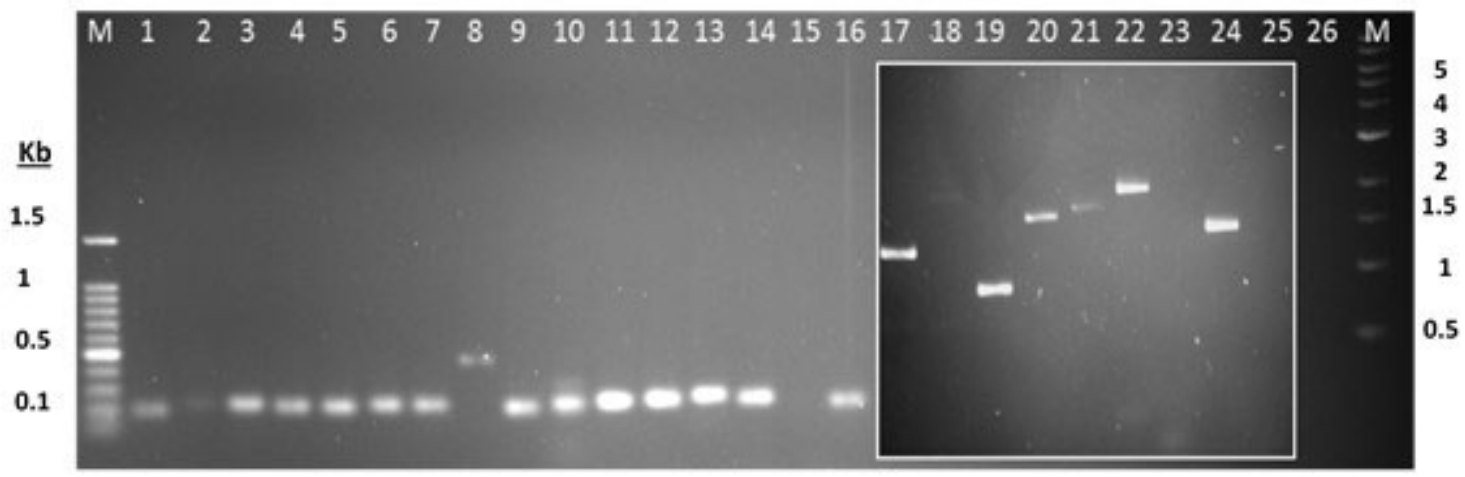


Fig. S3
$\mathrm{GM}-\mathrm{NH}_{3}+\mathrm{L}$ ala
$\mathrm{GM}-\mathrm{NH}_{3}+\mathrm{D}$ ala
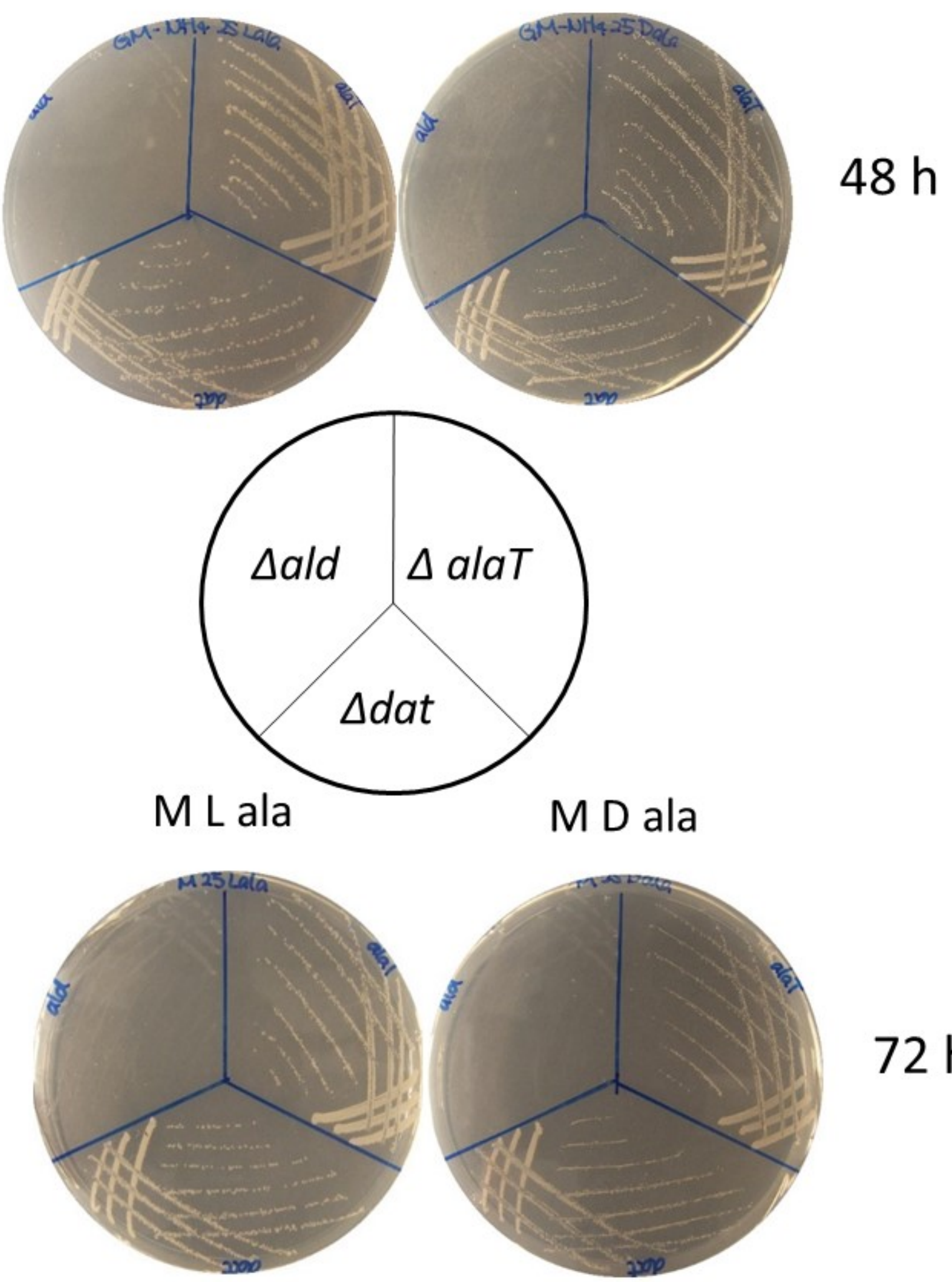

$72 \mathrm{~h}$ 